\title{
HIF-stabilization prevents delayed fracture healing
}

2 One Sentence Summary: We here provide evidence for a promising preventive approach to enhance bone

3 regeneration capacities and potentially to overcome compromised bone healing conditions by combining

$4 \quad$ DFO and MIF - as potent HIF-stabilizers.

5

6 Authors: Annemarie Lang ${ }^{1,2,3}$, Sarah Helfmeier ${ }^{1}$, Jonathan Stefanowski ${ }^{1,2}$, Aditi Kuppe ${ }^{1}$, Vikram 7 Sunkara ${ }^{4,5}$, Moritz Pfeiffenberger ${ }^{1,2}$, Angelique Wolter ${ }^{1,2}$, Alexandra Damerau ${ }^{1,2}$, Shabnam Hemmati8 Sadeghi ${ }^{1,3,6}$, Jochen Ringe ${ }^{1,3}$, Rainer Haag ${ }^{6}$, Anja E. Hauser ${ }^{1,2}$, Max Löhning ${ }^{1,2}$, Carsten Perka ${ }^{7}$, Georg N. 9 Duda $^{3,7,8 *}$, Paula Hoff ${ }^{1,2,3}$, Katharina Schmidt-Bleek ${ }^{3,7,8 \dagger}$, Timo Gaber ${ }^{1,2,3 \uparrow *}$, Frank Buttgereit ${ }^{1,2,3 \dagger}$

$11 \uparrow$ These authors contributed equally (KSB, TG, FB).

12 *To whom correspondence should be addressed: timo.gaber@charite.de; georg.duda@charite.de

\section{Affiliations:}

$15{ }^{1}$ Charité - Universitätsmedizin Berlin, corporate member of Freie Universität Berlin, Humboldt-Universität 16 zu Berlin, and Berlin Institute of Health, Department of Rheumatology and Clinical Immunology, Berlin, 17 Germany

$18{ }^{2}$ German Rheumatism Research Centre (DRFZ) Berlin, a Leibniz Institute, Berlin, Germany

$19{ }^{3}$ Charité - Universitätsmedizin Berlin, corporate member of Freie Universität Berlin, Humboldt-Universität 20 zu Berlin, and Berlin Institute of Health, Berlin Institute of Health Center for Regenerative Therapies, 21 Berlin, Germany

$22{ }^{4}$ Computational Medicine, Zuse Institute Berlin, Berlin, Germany

$23{ }^{5}$ Department of Mathematics and Computer Science, Freie Universität Berlin, Berlin, Germany

$24 \quad{ }^{6}$ Institute of Chemistry and Biochemistry, Freie Universität Berlin, Berlin, Germany

$25{ }^{7}$ Charité - Universitätsmedizin Berlin, corporate member of Freie Universität Berlin, Humboldt-Universität zu Berlin, and Berlin Institute of Health, Center for Musculoskeletal Surgery, Berlin, Germany

$27{ }^{8}$ Charité - Universitätsmedizin Berlin, corporate member of Freie Universität Berlin, Humboldt-Universität zu Berlin, and Berlin Institute of Health, Julius Wolff Institute, Berlin, Germany 


\section{$31 \quad$ Abstract}

32 The initial phase of fracture healing decides on success of bone regeneration and is characterized by

33 an inflammatory milieu and low oxygen tension (hypoxia). Negative interference with or prolongation of

34 this fine-tuned initiation phase will ultimately lead to a delayed or incomplete healing such as non-unions

35 which then requires an effective and gentle therapeutic intervention. Common reasons include a

36 dysregulated immune response, immunosuppression or a failure in cellular adaptation to the inflammatory

37 hypoxic milieu of the fracture gap and a reduction in vascularizing capacity by environmental noxious

38 agents (e.g. rheumatoid arthritis, smoking). The hypoxia-inducible factor (HIF)-1 $\alpha$ is responsible for the

39 cellular adaptation to hypoxia, activating angiogenesis and supporting cell attraction and migration to the

40 fracture gap. Here, we hypothesized that stabilizing HIF-1 $\alpha$ could be a cost-effective and low-risk

41 prevention strategy of fracture healing disorders. Therefore, we combined a well-known HIF-stabilizer -

42 deferoxamine (DFO) - and a less known HIF-enhancer - macrophage migration inhibitory factor (MIF) -

43 to synergistically induce improved fracture healing. Stabilization of HIF-1 $\alpha$ enhanced calcification and

44 osteogenic differentiation of MSCs in vitro. In vivo, the application of DFO with or without MIF during the

45 initial healing phase accelerated callus mineralization and vessel formation in a clinically relevant mouse-

46 osteotomy-model in a compromised healing setting. Our findings provide support for a promising preventive

47 strategy towards bone healing disorders in patients with a higher risk due to e.g. delayed neovascularization

48 by accelerating fracture healing using DFO and MIF to stabilize HIF-1 $\alpha$. 


\section{$50 \quad$ Introduction}

Fracture healing combines temporal and spatial fine-tuned and tightly regulated regenerative

52 processes, which lead to a complete restoration of the broken bone without scar formation. However, a

53 minimum of $10 \%$ of patients with fractures suffer from fracture healing disorders such as delayed or

54 incomplete healing (non-unions) leading to immobility, pain, a loss in quality of life, and generate an

55 economic burden for the society $(1,2)$. While trauma severity and location can determine the healing success

56 (3), several risk factors have been described to potentially impair the fracture healing process such as age

57 and lifestyle including obesity, alcohol abuse, and smoking (4). Of note, smoking is supposed to reduce

58 vessel formation and to stimulate adverse immune reactions (5). Furthermore, chronic inflammatory

59 diseases, such as rheumatoid arthritis (RA) or systemic lupus erythematosus, have been related to fracture

60 healing disorders (6-8). Patients with fracture healing disorders often require several further revision

61 surgeries. Apart from surgical intervention, local delivery of recombinant human (rh)BMP-2 into the

62 fracture gap has been demonstrated in clinical studies to be effective $(9,10)$. However, several adverse

63 effects in humans strongly restrict the clinical implementation of this approach (11) and alternative strategies

64 or preventive measures are lacking.

65 If a fracture is stabilized such that inter-fragmentary movements can occur, secondary bone healing

66 is initiated leading to bone regeneration via an endochondral ossification process bridging the fracture gap.

67 Endochondral bone healing can be divided into five phases: i) initial pro-inflammatory phase, ii) anti-

68 inflammatory phase; iii) fibrocartilaginous or soft callus phase; iv) woven bone or hard callus phase; and v)

69 the remodeling phase leading to the bone restitution in form and function according to the mechanical strain

70 (12). The shift from pro- to anti-inflammatory phase is a requirement for angiogenic and pro-osteogenic

71 processes during the initial phase of fracture healing and determine the subsequent regeneration cascades

$72(6,13)$. This shift is essential for the initiation of bone reconstruction involving the recruitment of i)

73 monocytes/macrophages, clearing the inflammatory scene and paving the path for revascularization, ii) pre-

74 osteoblasts and mesenchymal stromal cells (MSCs) as basic component of bone reconstruction, iii)

75 fibroblasts, which are required for early callus formation and bone matrix formation, and iv) endothelial 
cells (ECs) for neovascularization $(14,15)$. Crucial elements for successful bone healing are balanced control and termination of the pro-inflammatory cascade (16), proper mesenchymal differentiation and cartilage formation, controlled invasion of vessels (17) as well as a sufficient mechanical stabilization (1821).

The hypoxic and inflammatory conditions in the fracture hematoma result from the disruption of vessels, accumulation of inflammatory cells, increased cell death of e.g. erythrocytes and the lack of nutrients, oxygen, high lactate and low $\mathrm{pH}$ - a cytotoxic environment which has to be down-regulated to maintain regenerative cells. The oxygen tension within the fracture site is reduced over the first week after trauma being accompanied by a reduction (50\%) of blood flow (22-27). Therefore, cellular adaptation mechanisms towards hypoxia are strongly activated - such as the hypoxia inducible factor (HIF) signaling pathway. Both, HIF-1 and HIF-2 are essential for cells to survive hypoxic conditions and to aim at increasing the oxygen supply while reducing oxygen consumption (28). While HIF-1 $\beta$ is constitutively expressed, HIF$1 \alpha$ is oxygen-dependently activated and stabilized at less than $5 \%$ oxygen (29). HIF-1 $\alpha$ is then translocated to the nucleus, where it heterodimerizes with HIF-1 $\beta$, binds to its target sequences (hypoxia-responsive

90 elements), and activates genes necessary for cellular hypoxic adaptation (29, 30). Under normoxic

91 conditions, HIF-1 $\alpha$ is hydroxylized by the oxygen- and iron-dependent prolyl-hydroxylase domain (PHD)

92 enzyme/protein and degraded by cellular proteasomes.

93 We have previously examined fracture hematomas obtained from immunologically restricted patients 94 and found reduced osteogenic differentiation due to reduced runt-related transcription factor 2 (RUNX2) 95 expression, exaggerated immune reactions (interleukin IL-8, C-X-C chemokine receptor type 4 CXCR4), 96 and high expression of HIFIA but inadequate expression of target genes (31). We also found higher numbers 97 of monocytes/macrophages, natural killer T (NKT) cells and activated T cells within fracture hematomas of 98 immunologically restricted patients accompanied by higher levels of IL-6, IL-8, tumor necrosis factor 99 (TNF) $\alpha$ and chemokines (e.g. Eotaxin) (32). In order to increase target gene expression, HIF-1 $\alpha$ can be 100 chemically stabilized by different factors, which either inhibit the $\mathrm{O}_{2}$-sensing PHD such as deferoxamine 
101 (DFO) or directly interfere with the downstream effects after translocation to the nucleus e.g. the 102 macrophage-migration inhibitory factor (MIF) $(33,34)$.

103 Here, we initially performed a single center retrospective study to investigate the risk factors for

104 fracture healing disorders in a Charité-located patient cohort and to determine the clinical need to 105 preventively support and accelerate fracture healing. Furthermore, we comprehensively systematically 106 reviewed the available literature to delineate the potential of our drafted therapeutic approach of promoting

107 fracture healing using DFO. To this end, we summarized several studies which demonstrated the efficacy

108 of DFO to promote bone fracture healing in a variety of animal models (mouse, rat, rabbit) focusing on

109 different kinds of bone defects. Experimentally, we conducted in vitro studies on osteogenesis, which

110 supported the enhancing effect of HIF-1 $\alpha$ stabilization on osteogenic differentiation of MSCs and its

111 counteracting efficacy against e.g. glucocorticoid (GC)-induced inhibition of osteogenesis. Moreover, we

112 tested the combination of MIF and DFO in a mouse-osteotomy-model of compromised bone healing

113 conditions in order to evaluate their preventive capability to counteract delayed bone healing in this

114 clinically relevant model. Thus, our study provides evidence for a promising preventive strategy to

115 accelerate fracture healing by applying potent HIF-stabilizers during initial fracture treatment in patients at

116 risk that may finally help to minimize bone healing disorders. 
119 Results

\section{Fracture healing disorders in a single-center patient cohort -A retrospective study}

Fracture healing disorders are associated with the incidence of several different risk factors e.g. age,

122 gender, fracture location, comorbidities of medications. Therefore, we investigated the main risk factors for

123 fracture healing disorders in a single-center retrospective study at the Center for Musculoskeletal Surgery,

124 Charité-Universitätsmedizin Berlin to get a more precise view of the patient's need for a therapeutic support

125 and acceleration of fracture healing. To this end, we screened data from inpatients treated in the hospital

126 during 2012 (fig. S1). Finally, 79 cases fulfilling inclusion criteria were included in the study (table S1), as

127 well as 178 controls matched for age and fracture location while patients aged $<18$ years, having open

128 fractures or metastases close to the fracture location were excluded.

129 First, we performed a descriptive statistical analysis to compare the two groups based on the collected

130 parameters such as body mass index (BMI), gender, alcohol abuse (repeated consumption of alcohol per

131 week), smoking, glucocorticoid and NSAID treatment as well as diagnosed comorbidities as RA,

132 osteoporosis, arterial hypertension and diabetes type 2 (table S2). The average BMI of the two groups was

133 similar. Comparing the control and case group, we found more male patients (53.2\% vs. 44.9\%) than female

134 patients $(46.8 \%$ vs. $55.1 \%)$ to be affected by fracture healing disorders. Interestingly, alcohol abuse was

135 more often present in the control group as compared to the case group (14.4\% vs $2.7 \%$ ) which was the

136 opposite for smoking ( $28.1 \%$ vs. $37.8 \%$ ). Regarding medications, a higher number of patients with fracture

137 healing disorders were continuously treated with glucocorticoids (6.3\% vs. $1.6 \%)$ and NSAIDs (7.6\% vs.

$1382.7 \%$ ) as compared to controls. Although the frequencies of osteoporosis and diabetes type 2 were

139 comparable within both groups, the incidences of RA (6.3\% vs. $0.5 \%)$ and arterial hypertension (43\% vs.

$14033.5 \%$ ) were higher in the case group. To select potentially relevant factors, an univariable logistic

141 regression was performed (Table 1) using a significance level of 0.15 resulting in the selection of smoking,

142 RA and arterial hypertension for detailed analysis via multivariable logistic regression. Age and gender,

143 parameters well-known to be associated with a poor fracture healing outcome, were additionally included

144 in the subsequent multivariable logistic regression. Statistical analysis using multivariable logistic 
145 regression showed a high significance for RA $(P=0.028)$ and a trend for smoking $(P=0.075)$ to be 146 associated with fracture healing disorders such as non-unions.

147 Table 1: Univariable and multivariable logistic regression.

\begin{tabular}{|c|c|c|c|c|c|c|}
\hline & \multicolumn{2}{|c|}{ Univariable } & \multicolumn{4}{|c|}{ Multivariable } \\
\hline & $\begin{array}{c}\text { Omnibus } \\
\text { test }\end{array}$ & $P$-value & 5 & 4 & 3 & 2 \\
\hline Age & 0.597 & $0.597 *$ & 0.118 & 0.202 & - & - \\
\hline Gender & 0.219 & $0.219^{*}$ & 0.148 & - & - & - \\
\hline BMI & 0.212 & - & & & & \\
\hline Alcoholism & 0.657 & - & & & & \\
\hline Smoking & 0.131 & 0.129 & 0.065 & 0.063 & 0.048 & 0.075 \\
\hline $\begin{array}{l}\text { Rheumatoid } \\
\text { Arthritis }\end{array}$ & 0.006 & 0.022 & 0.057 & 0.047 & 0.040 & 0.028 \\
\hline Glucocorticoids & 0.725 & - & & & & \\
\hline NSAIDs & 0.336 & - & & & & \\
\hline Osteoporosis & 0.813 & - & & & & \\
\hline $\begin{array}{l}\text { Arterial } \\
\text { Hypertension }\end{array}$ & 0.143 & 0.142 & 0.085 & 0.058 & 0.137 & - \\
\hline Diabetes Type 2 & 0.908 & - & & & & \\
\hline
\end{tabular}

We have previously found that immunologically restricted patients (including e.g. autoimmune 150 disease) show a disturbed response to hypoxia in the fracture hematoma (31). Therefore, we hypothesized 151 that HIF-stabilization can accelerate fracture healing in those patients. The inhibition of iron-dependent 152 PHDs by iron chelators or competitors activates HIF-mediated pathways such as angiogenesis and 153 osteogenesis. The iron chelator DFO is well-known from in vitro and in vivo studies to stabilize HIF (35, 15436 ).

Given this background, we performed a systematic literature review to delineate existing preclinical studies on the effectiveness and efficiency of DFO in fracture healing. In detail, we asked the question whether the local application of DFO in the fracture gap enhanced bone formation ( $\mu \mathrm{CT}$; histomorphometry)

158 during fracture healing in animal models with normal or disturbed fractures of long-bones or Ossa 159 irregularia (mandibula or zygomatic arch). The complete search strategy can be found in figure S2 and S3. 
160 We included 20 studies for a descriptive analysis. A meta-analysis was not applicable due to the variability

161 of studies, the variety of models and the information provided (e.g. data values not given; confidential

162 intervals not indicated). Nevertheless, all included studies demonstrated the efficacy of DFO to promote

163 bone fracture healing in a variety of animal models (mouse, rat, rabbit) with different bone defects (Table

164 2). In these studies, DFO was applied in varying doses (in mouse: $20 \mu 1$ of $200 \mu \mathrm{M}-400 \mu \mathrm{M}$ ) either once

165 or repetitively by local injection directly into the bone defect/gap or by loading onto a scaffold implanted

166 into the bone defect/gap to counteract a delayed healing or non-union. DFO treatment resulted in a strong

167 promotion of angiogenesis/vessel formation and bone regeneration independent of the species, model and

168 evaluation methods (22-39).

Table 2: Systematic literature review on the potential of DFO to accelerate bone formation/healing.

\begin{tabular}{|c|c|c|c|c|c|}
\hline$\frac{\mathscr{e}}{\mathscr{E}}$ & $\stackrel{\mathscr{E}}{\mathscr{0}}$ & Model & $\begin{array}{l}\text { Application Route \& } \\
\text { Concentration }\end{array}$ & $\begin{array}{l}\text { Bone/vessel } \\
\text { formation }\end{array}$ & Refs. \\
\hline \multirow{8}{*}{ 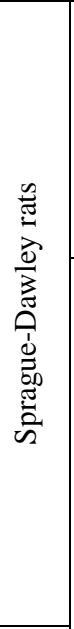 } & \multirow{3}{*}{ 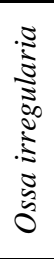 } & $\begin{array}{c}\text { Mandibular distraction } \\
\text { osteogenesis }\end{array}$ & $\begin{array}{l}\text { Local injection every other day } \\
\text { (5 doses); DFO }=200 \mu \mathrm{M}\end{array}$ & +++ & $(37-39)$ \\
\hline & & $\begin{array}{c}\text { Mandibular osteotomy or } \\
\text { distraction }+ \\
\text { Radiation }\end{array}$ & $\begin{array}{l}\text { Local injection every other day } \\
\quad(5 \text { doses }) ; \mathrm{DFO}=200 \mu \mathrm{M}\end{array}$ & +++ & $(40-46)$ \\
\hline & & $\begin{array}{c}\text { Zygomatic arch critical-size } \\
\text { bone defect }\end{array}$ & $\begin{array}{l}\text { Local injection every other day } \\
(>20 \text { doses }) ; \mathrm{DFO}=200 \mu \mathrm{M}\end{array}$ & +++ & $(47)$ \\
\hline & \multirow{9}{*}{ 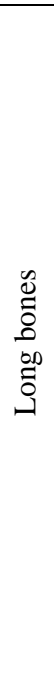 } & $\begin{array}{l}\text { Segmental femur defects } \\
\text { (wire) }\end{array}$ & $\begin{array}{c}\text { Application onto scaffold; } \\
\text { DFO }=400 \mu \mathrm{M}\end{array}$ & +++ & $(48)$ \\
\hline & & Tibial non-union model & $\begin{array}{c}\text { Application onto scaffold; } \\
\mathrm{DFO}=1 \mathrm{mg} / \mathrm{kg}\end{array}$ & +++ & $(49)$ \\
\hline & & Femoral drilling hole model & $\begin{array}{l}\text { Application onto scaffold; } \\
\text { DFO }=1 \mathrm{mg} / \mathrm{ml}\end{array}$ & +++ & $(36)$ \\
\hline & & $\begin{array}{l}\text { Tibia cortical drilling + } \\
\text { hindlimb unloading }\end{array}$ & $\begin{array}{l}\text { Local injection every other day } \\
(2-5 \text { doses }) ; \mathrm{DFO}=200 \mu \mathrm{mol} / \mathrm{l}\end{array}$ & +++ & $(50)$ \\
\hline & & $\begin{array}{l}\text { Femoral drilling hole } \\
+ \text { ovariectomy }\end{array}$ & $\begin{array}{c}\text { Application onto scaffold; } \\
\text { DFO }=2 \mu \mathrm{g}\end{array}$ & +++ & $(51)$ \\
\hline \multirow{2}{*}{ 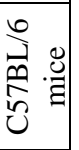 } & & $\begin{array}{l}\text { Distraction osteogenesis } \\
\text { in tibia (plate) }\end{array}$ & $\begin{array}{c}\text { Local injection every other day } \\
(6 \text { doses }) ; \text { DFO }=200 \mu \mathrm{M}\end{array}$ & +++ & $(35)$ \\
\hline & & $\begin{array}{c}\text { Stabilized femur } \\
\text { fracture model (pin) }\end{array}$ & $\begin{array}{l}\text { Local injection every other day } \\
(5 \text { doses }) ; \mathrm{DFO}=200 \mu \mathrm{M}\end{array}$ & +++ & $(52)$ \\
\hline \multirow{2}{*}{$\begin{array}{c}3 \\
\mathrm{z} \\
\mathrm{z}\end{array}$} & & Segmental radius bone defects & $\begin{array}{l}\text { Application onto scaffold } \\
\text { DFO }=2 \mathrm{mM}\end{array}$ & +++ & (53) \\
\hline & & Mid-shaft ulnar defect & $\begin{array}{c}\text { Application onto scaffold } \\
\mathrm{DFO}=200 \mu \mathrm{M}\end{array}$ & +++ & $(54)$ \\
\hline
\end{tabular}


173 increased by combination with MIF which we have shown to further enhance HIF activity, especially in

174 immune cells and endothelial cells (55).

\section{Combining DFO and MIF to enhance in vitro calcification of hMSCS}

To evaluate the potential of MIF and DFO as enhancer of bone formation in vitro, different concentrations alone and in combination were tested in an osteogenic differentiation assay of bone marrow derived human (h)MSCs. High concentrations of Dexamethasone (Dex; $10^{-3} \mathrm{M}$ ) were used as a technical in vitro model to strongly induce delayed calcification while $10^{-8} \mathrm{M}$ Dex was the respective control which is usually included in the osteogenic medium $(\mathrm{OM})$. Large differences in calcification were observed after 4 weeks under normoxic conditions and used further titration experiments (fig. S4; fig. S5; Fig. 1). Normoxic conditions represented the inadequate adaptation to hypoxia as mentioned before. Varying concentrations of MIF and DFO alone and in combination were examined for their effect on hMSC calcification. The application of DFO alone showed significant increases in calcification after 4 weeks at 62.5, 125, 250 and $500 \mu \mathrm{M}$ which was also observed at 250 and $500 \mathrm{ng} / \mathrm{ml}$ MIF (fig. S5A, B). A double normalization to both controls $\left(10^{-3} \mathrm{M}\right.$ and $10^{-8} \mathrm{M}$ Dex) revealed that MIF alone did not enhance calcification (Fig. 1A), while DFO alone already showed mean increases between $43 \%$ - 45.9\% (Fig. 1B). The combination of MIF and DFO led to a significant increase in calcium deposition when using $50 \mathrm{ng} / \mathrm{ml} \mathrm{MIF}+125 \mu \mathrm{M}$ DFO and 100 ng/ml MIF $+125 \mu \mathrm{M}$ DFO with a mean increase of $61.7 \%$ to $86.7 \%$ for both groups (fig. S5C; Fig. 1C).
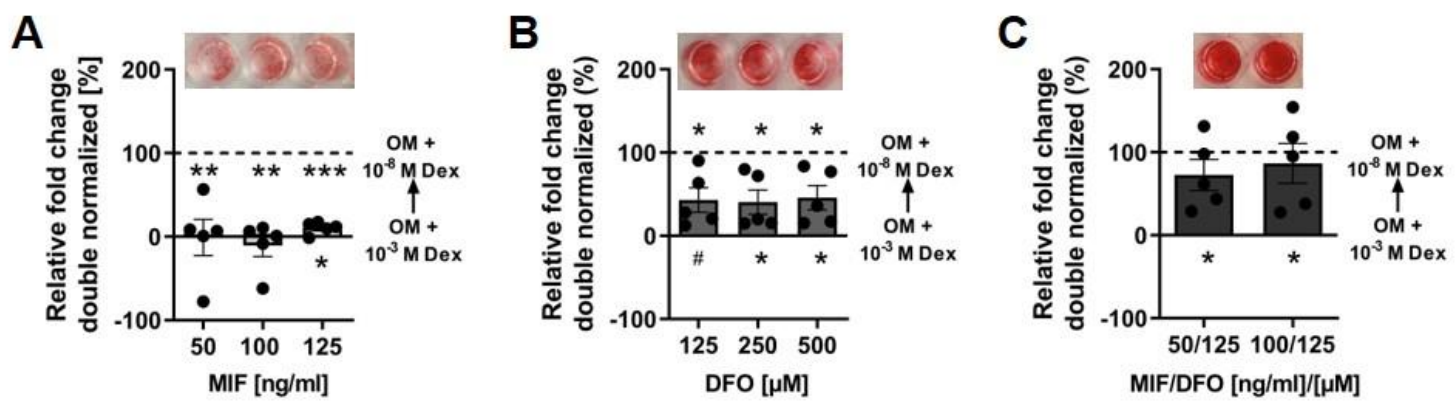

Figure 1: In vitro studies on the effect of MIF and DFO on hMSC calcification. Double normalization of OD values (Dex $10^{-3} \mathrm{M}=0 \%$; Dex $10^{-8} \mathrm{M}=100 \%$ ) for selected MIF (A), DFO (B) and MIF/DFO (C) concentrations. Asterisks above and below the bars indicate significant differences as compared to the respective control. Bar graphs show mean \pm SEM and individual data points. One sample t-test was used to determine the statistical significance; $P$-values are indicated with ${ }^{\#} P<0.07 ; * P<0.05 ; * * P<0.01 ; * * * P<0.001$. Exemplary images of Alizarin red staining in 96 well are displayed before quantification. 


\section{The effect of MIF and DFO on in vivo bone formation in a delayed healing model}

Based on the results from the systematic review and our in vitro experiments, we tested MIF and DFO, both alone and in combination in an experimental delayed healing model using a modified mouseosteotomy which results in a local blockage of angiogenesis and results in a delayed bone healing due to insertion of an absorbable bovine Col-I scaffold (ACS) in the osteotomy gap (56). We previously demonstrated that this model features a disturbance in cell invasion, vessel formation and consecutively bone formation when compared to empty-gap controls at 2 and at 3 weeks after osteotomy (56). The osteotomy gap $(0.7 \mathrm{~mm})$ was introduced in the femur of 12 weeks old female C57BL/6N mice. A stable fixation of the osteotomized bone was obtained using an external fixator.

Bone formation was significantly intensified in the DFO and MIF/DFO treated groups as measured observed increased levels in total mineralized bone tissue in the fracture area and in the fracture gap in all treatments groups after 2 weeks (Fig. 2C). Interestingly, MIF also significantly induced total bone formation especially in the fracture gap at 2 weeks when compared to the corresponding ACS control, while in the other groups mineralized bone formation was more pronounced within the gap (Fig. 2C). Of note, we observed a higher cartilage content in the MIF and DFO group at 2 weeks as compared to the ACS control, while MIF/DFO exhibited similar amounts of cartilage. These differences were not present at 3 weeks, since animals with only ACS show a delayed endochondral ossification (between week 2 and 3; fig. S6C) (56).

216 However, the complete bridging of the fracture gap with cartilage and mineralized bone was observed in $21750 \%$ of DFO and MIF/DFO mice additionally indicating an acceleration of the endochondral ossification 218 process (fig. S7). Interestingly, bridging between cortices was more often observable in the MIF/DFO 219 treated group (50\%) (fig. S7). Finally, DFO and MIF/DFO led to a higher recruitment of osterix (Osx) $)^{+}$ osteoprogenitors/osteoblasts to the fracture gap at 2 weeks (Fig. 2D, E). In addition, Osx ${ }^{+}$cells were more 
223 in our delayed healing mouse-osteotomy-model.

A
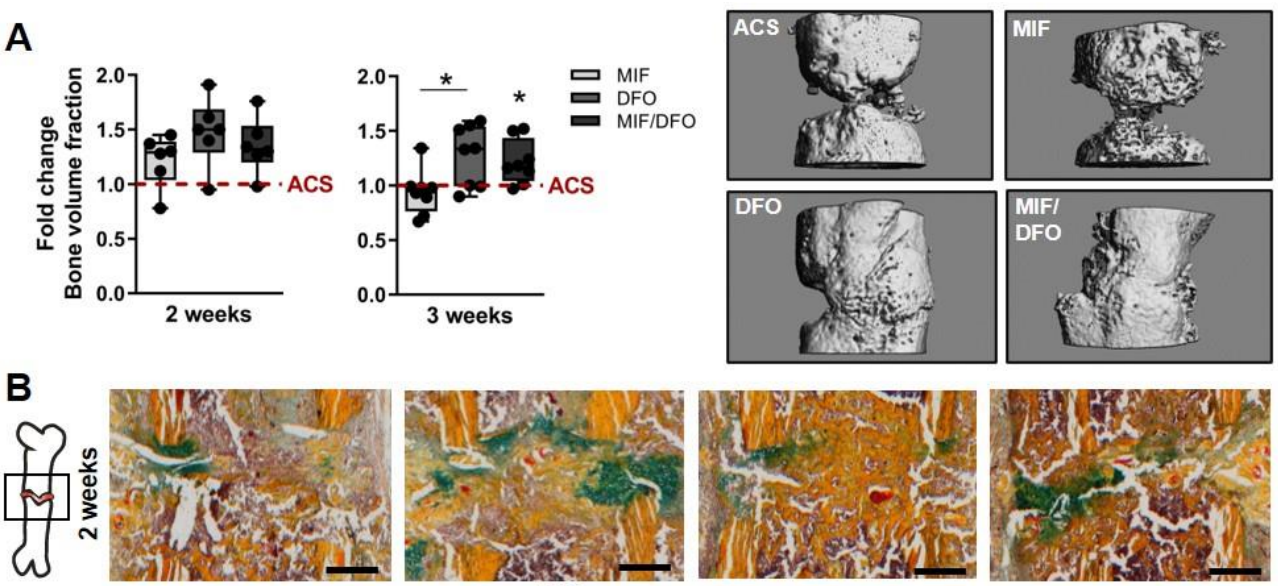

DFO

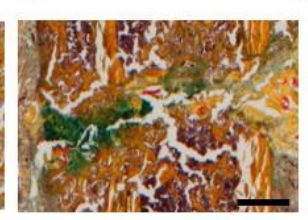

C
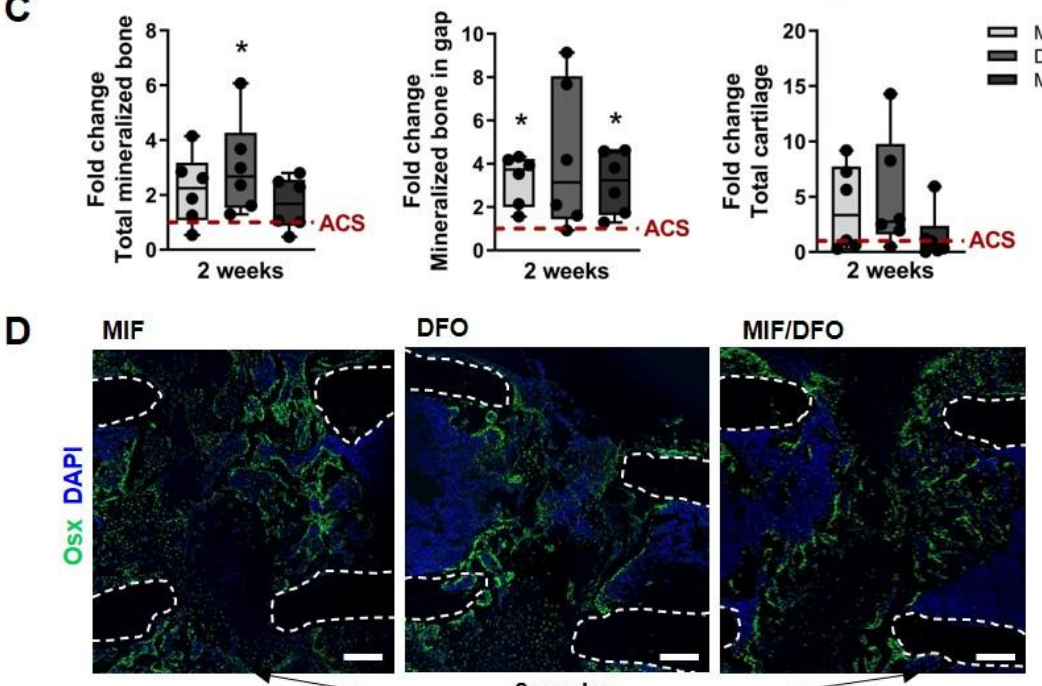

E

Figure 2: Bone regeneration in a delayed healing model after single dose of MIF or/and DFO. (A) MicroCT quantification at 2 weeks and at 3 weeks post-osteotomy normalized to the median of the ACS group (indicated as dotted line $=1$ ). Bone volume fraction = bone volume/callus volume. Representative $3 D$ microCT reconstructions at week 3. (B) Representative images of Movat's pentachrome staining for each group at week 2. yellow - mineralized bone/scaffold; green - cartilage; magenta - bone marrow. (C) Histomorphometry of Movat's pentachrome staining using ImageJ. Data were normalized to the median of the ACS group (indicated as dotted line $=1$ ). (D) Representative images of immunofluorescence staining of Osterix (Osx) and its quantification. White dotted lines indicate cortices.

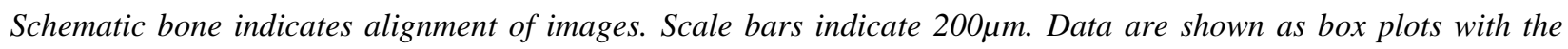
median as horizontal line, interquartile range as boxes, minimum/maximum as whiskers and individual data points. Wilcoxon signed rank test was applied to determine difference against the ACS control group (hypothetical value $=1$ ) and Kruskal Wallis test with Dunn's multiple comparison test was used to compare groups. ${ }^{\#} P<0.07 ;{ }^{*} P<0.05$. 


\section{Vessel formation is increased by HIF-stabilization}

Revascularization is crucial for bone regeneration, tightly regulated by the microenvironment and appears in two waves at day 7 and day $21(17,57)$ CD $31^{+}$endothelial progenitors enter the fracture gap

239 during the initial phase of fracture healing (until day 7) (57). DFO is known to strongly promote

240 revascularization by the induction of vascular endothelial growth factor (VEGF) expression, a target gene 241 of HIF-1 $\alpha$ (34). Therefore, we analyzed the osteotomy site in our delayed healing model for the presence of

$242 \mathrm{CD} 1^{+} \mathrm{Emcn}^{+}$vessels. At week 2 and 3, we found significantly more CD31 ${ }^{+} \mathrm{mcn}^{+}$vessels in the fracture 243 gap of the treatment groups compared to the ACS control group while cell invasion was more pronounced

244 at week 2 and comparable to the ACS control group at week 3 (Fig. 3A-C). Pixel intensity analysis revealed

245 elevated Emcn and CD31 expressions in the DFO and MIF/DFO group at week 2 indicating a higher 246 appearance of $\mathrm{CD} 1^{+}$and $\mathrm{Emcn}^{+}$cells and a higher vascular formation (Fig. 3D-F). Interestingly, MIF alone 247 also induced the expression of CD31 in the fracture gap at week 2 compared to the ACS group (Fig. 3E). 248 However, expression levels were comparable between all groups at week 3. When examining earlier 249 timepoints (day 3 and 7), we observed a comparable appearance of CD31 ${ }^{+}$endothelial progenitors in all 250 groups but the DFO group (fig. S8A). In the DFO group, we observed a reduced number of CD31 251 endothelial progenitors at day 7, which was in line with the overall low cell number (DAPI) in the fracture 252 gap during the early stage (fig. S8A). Moreover, immunofluorescence images indicated a pronounced 253 invasion of $\mathrm{CD} 1^{+}$endothelial progenitors in the region adjacent to the fracture gap (fig. S8B). We conclude 254 from our data that MIF, DFO as well as the combined MIF/DFO enhanced and accelerated revascularization 255 to a considerably higher extent than the corresponding control group as seen at week 2 and 3. 

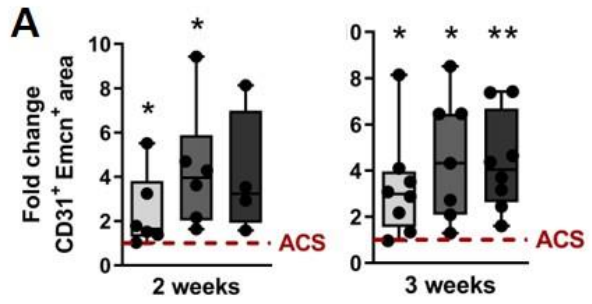

B
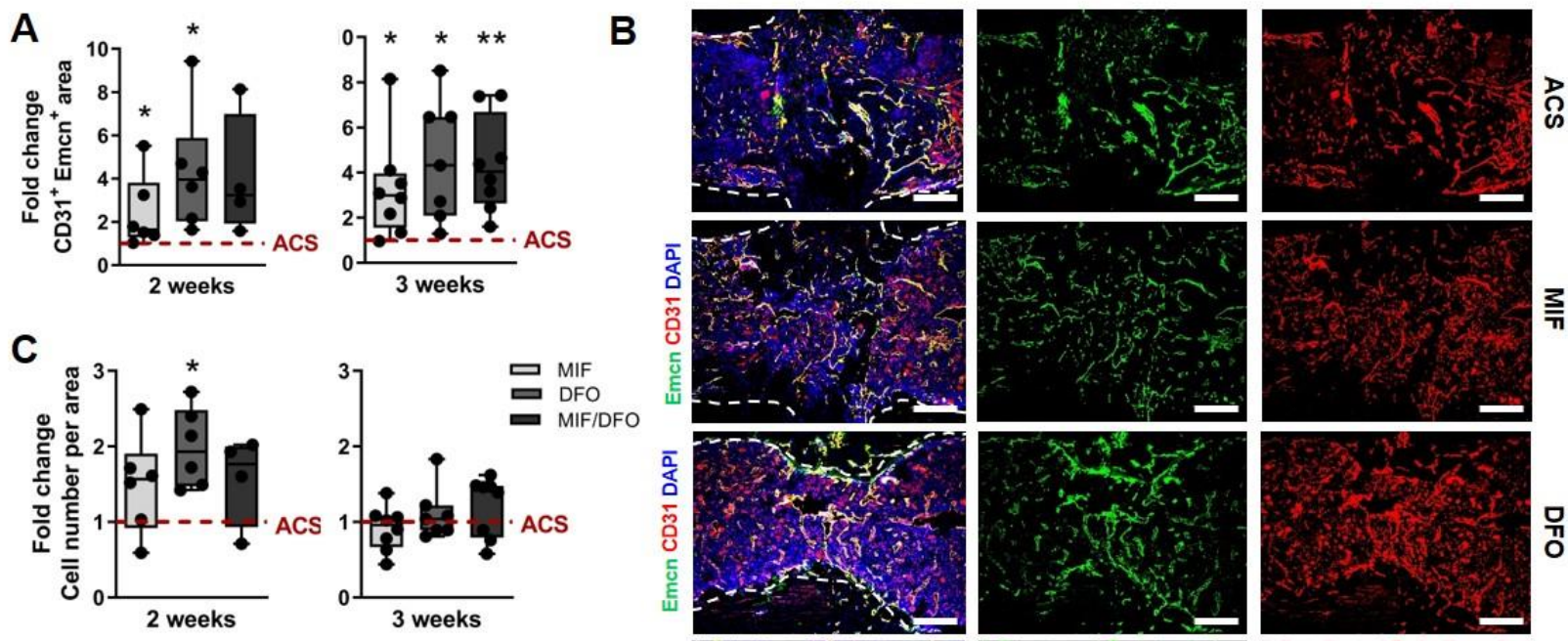

觉
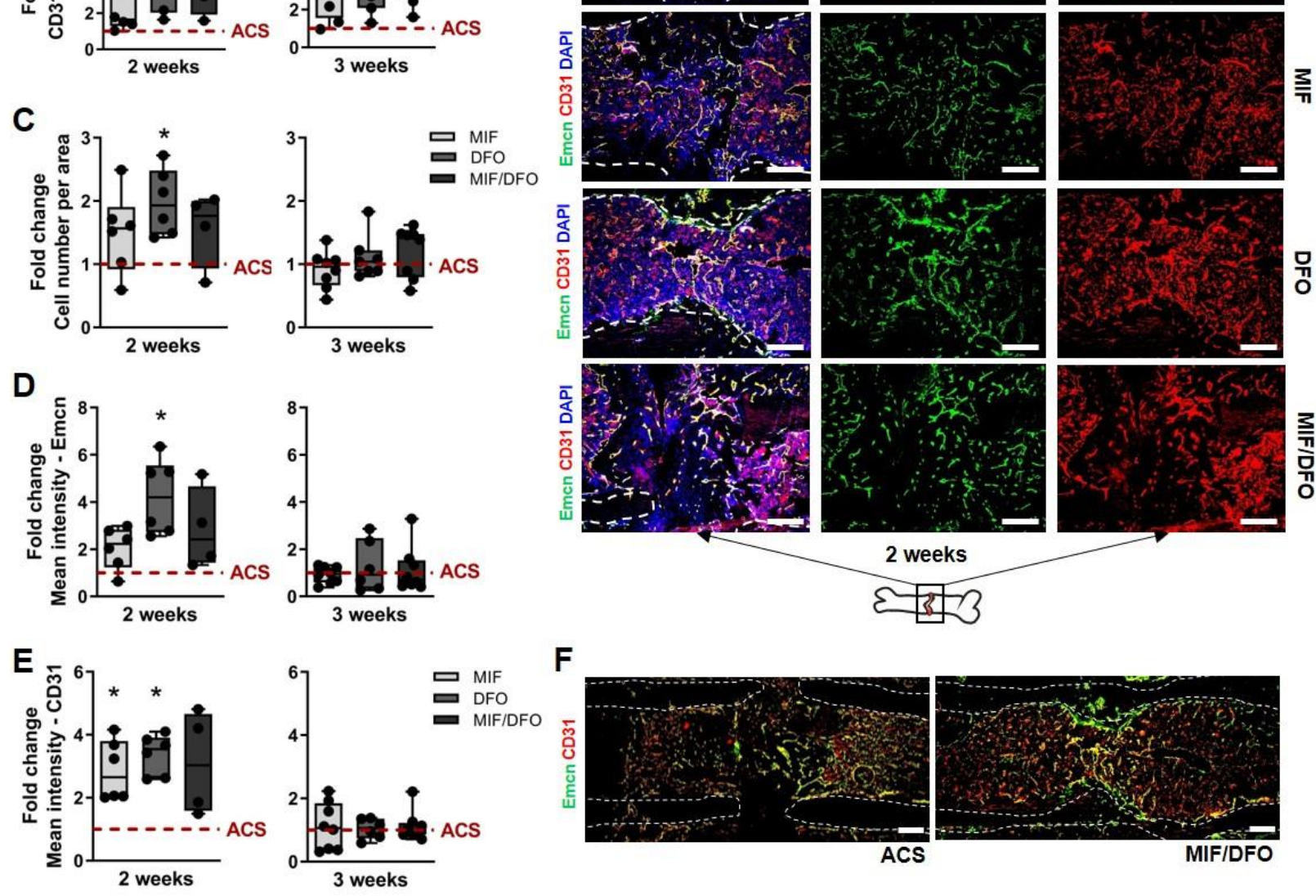

Figure 3: Revascularization in a delayed healing model under MIF, DFO and MIF/DFO treatment. (A-C) Quantified $C D 31^{+} \mathrm{Emcn}^{+}$stained areas $(A)$ and cell numbers per area $(C)$ normalized to the median of the ACS group (indicated as dotted line $=1)$ and $(B)$ corresponding representative images for week 2 and $3(N=6-8)$. $(D, E)$ Pixel based intensity analysis of Emcn $(D)$ and $C D 31(E)$ in the fracture gap normalized to the median of the ACS group (indicated as dotted line $=1$ ) and $(F)$ representative images of the combined staining for ACS and MIF/DFO at week 2. $(N=6-8)$. White dotted lines indicate cortices. Schematic bone indicates alignment of images. Scale bars $=200 \mu m$. Data are shown as box plots with the median as horizontal line, interquartile range as boxes, minimum/maximum as whiskers and individual data points. Wilcoxon signed rank test was applied to determine difference against the ACS control group (hypothetical value $=1$ ) and Kruskal Wallis test with Dunn's multiple comparison test was used to 


\section{DFO and MIF/DFO lead to enhanced presence of macrophages and TRAP+ cells}

Macrophages are essential during fracture healing and we have previously reported the crosstalk

270 between macrophages and vessel especially during the early phase $(56,57)$. Since the treatment with both

271 DFO and MIF/DFO resulted in an enhanced and accelerated endochondral ossification (Fig. 2) and

272 revascularization (Fig. 3), we further asked whether DFO and MIF/DFO contribute to an increased

273 macrophage invasion. Therefore, we analyzed the osteotomy area for the presence of F4/80+ cells at 3 days,

2747 days, 2 weeks and 3 weeks post-surgery. We observed a higher appearance of these cells in the DFO group

275 at day 3 indicating a faster recruitment to the fracture gap, while at day 7 the number was observably reduced

276 as compared to day 3 and the corresponding control (ACS) (Fig. 4A, B). No differences were found between

277 the other groups. Furthermore, we observed a more pronounced presence of tartrate-resistant acid

278 phosphatase (TRAP) $)^{+}$cells in the DFO and MIF/DFO group at week 2 than in the ACS group, which was

279 significantly reduced in the MIF group at week 3 when compared to the ACS and DFO group (Fig. 4C, D).

280 TRAP is a well-known marker of osteoclasts but can be also found on activated macrophages (58). In

281 addition, quantifications of the scaffold area after 2 and 3 weeks indicated a significant reduction of the

282 ACS in the osteotomy gap of both the DFO group (at week 2 and 3) and the MIF/DFO group (at week 2)

283 (fig. S9).

284 Together, these results suggest that both DFO and MIF/DFO promote recruitment of macrophages or 285 local proliferation during the early phase (day 3) and a pronounced expression of TRAP (osteoclast 286 differentiation) thereby supporting the bone regeneration process by providing the space for 287 revascularization. 

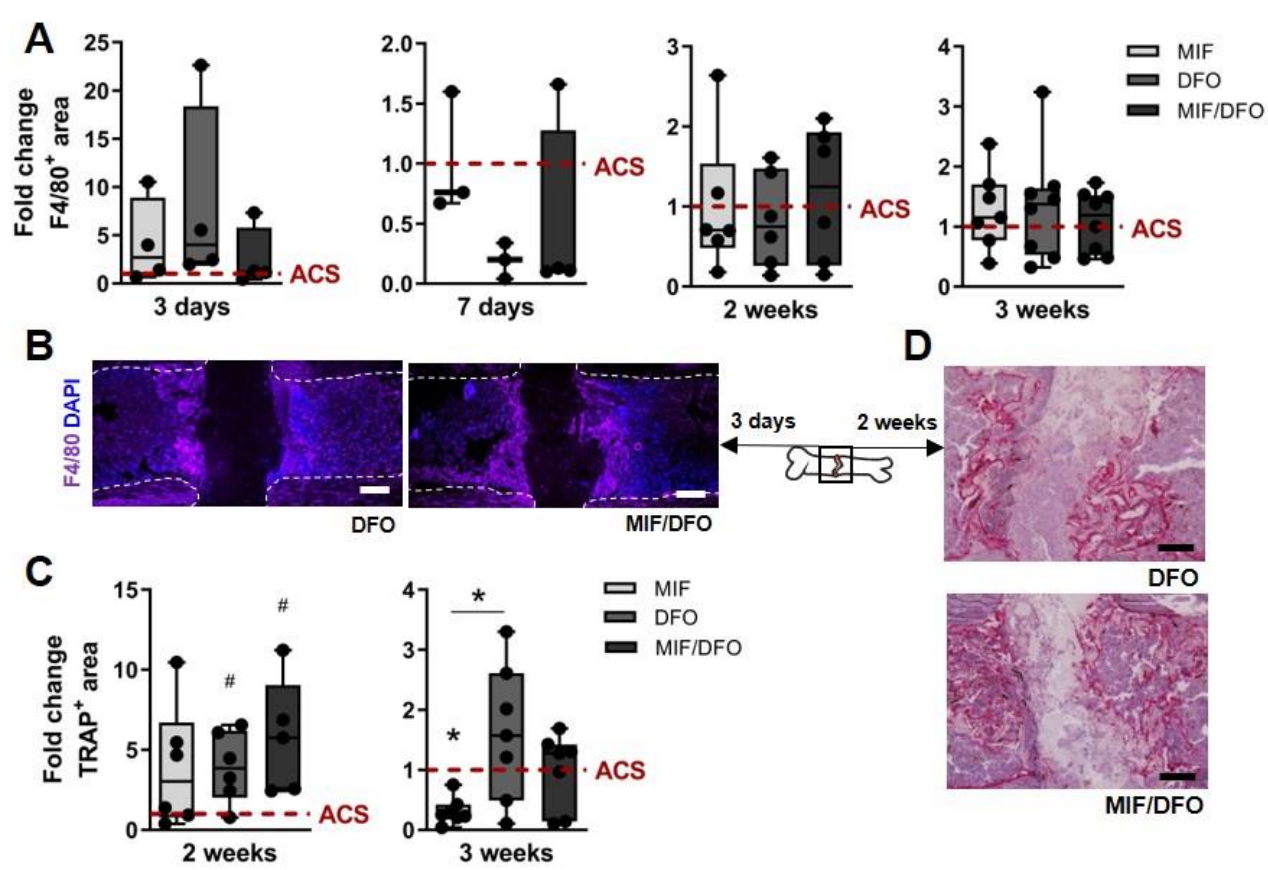

Figure 4: Presence of $\mathrm{F4} / 8 \mathrm{O}^{+}$macrophages and TRAP+ cells within the fracture gap. (A) Quantified $\mathrm{F} 4 / 80^{+}$area in the gap after 3, 7 days and 2, 3 weeks normalized to the median of the ACS group (indicated as dotted line =1; day 3, 7: $N=3-4$; week 2, 3: $N=6-8$ ). (B) Representative images for DFO and MIF/DFO at day 3. White dotted lines indicate cortices. Schematic bone indicates alignment of images. (C) Quantification of TRAP ${ }^{+}$area at week 2 and 3 normalized to the median of the ACS group (indicated as dotted line $=1$ ) and $(D)$ representative images. Scale bars $=200 \mu \mathrm{m}$. Data are shown as box plots with the median as horizontal line, interquartile range as boxes, minimum/maximum as whiskers and individual data points. Wilcoxon signed rank test was applied to determine difference against the ACS control group (hypothetical value $=1$ ) and Kruskal Wallis test with Dunn's multiple comparison test was used to compare groups. ${ }^{\#} P<0.07 ; * P<0.05$.

\section{Polyglycerol sulfate-based hydrogels as a potential releasing system for DFO}

Our results provide evidence for a beneficial effect of DFO in fracture healing and in combination

with MIF. In order to delineate a therapeutic option for fracture healing disorders based on HIF-stabilization

for e.g. patients with a delayed healing potential due to immunological and/or angiogenic constraints (31,

59), we evaluated the potential of an appropriate delivery/release system. Based on the observation that the

fracture hematoma obtained from immune-suppressed patients revealed an upregulated inflammatory profile during the initial phase of fracture healing, we tested dendritic polyglycerol sulfate (dPGS)-based polyethylene glycol-dicyclooctyne (PEG-DIC) hydrogels, which have been developed to act antiinflammatory (31, 32, 60-62). In addition, we used non-sulfated dendritic polyglycerol (dPG)-based PEG- 
310 compared to dPGS-based hydrogels over an observation span of 9 days (Fig. 5A). Supernatants from the 311 release assay were transferred to HEK 293 cells, and protein was collected after $24 \mathrm{~h}$. Western blot analysis

312 still indicated the functionality of the released DFO to stabilize HIF-1 $\alpha$ (Fig. 5B). hMSCs were co-cultivated

313 for 2 weeks with the DFO-loaded hydrogels and calcification was analyzed by Alizarin red staining (Fig.

314 5C, D). Unexpectedly, dPGS-based hydrogels seemed to have an inhibitory effect on in vitro hMSC

315 calcification independent of DFO loading. Thus, we concluded that the combination of DFO with dPG-

316 based hydrogels could be a beneficial approach regarding fracture healing. Moreover, a single dose 317 application/injection proved to be a promising alternative.

A
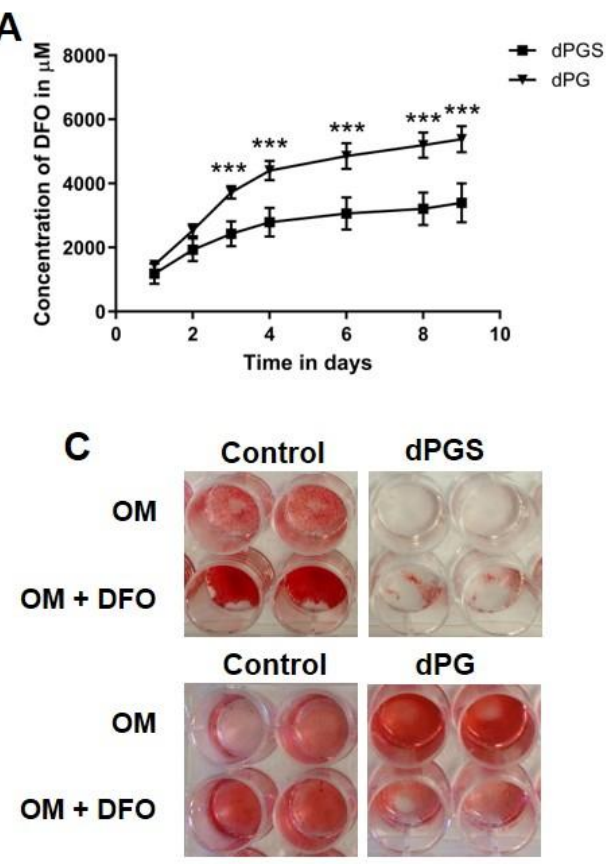

B

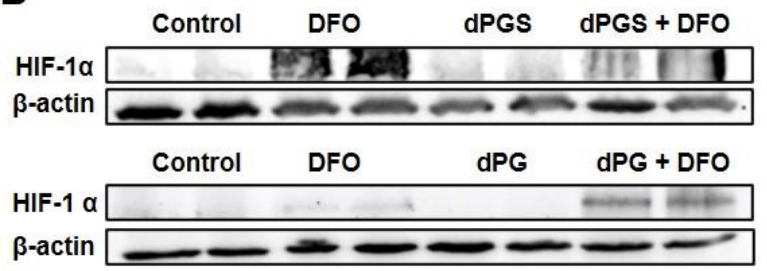

D

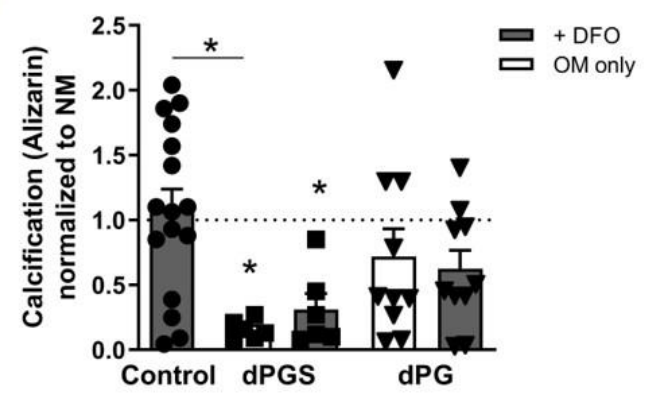

Figure 5: Release studies of DFO from a polyglycerol sulfate-based hydrogel (dPGS) and a polyglycerol-based =6). Two-way ANOVA with Bonferroni posttest was performed to determine significant differences. $* * * P<0.001$. (B) Western blot for HIF-1 $\alpha$ and $\beta$-actin from HEK 293 cells treated with supernatants from the release experiments. $(C, D)$ Alizarin staining was performed to determine the effect of the hydrogels and the released DFO on hMSC calcification at 2 weeks. $(N=6-11) . O M=$ osteogenic medium/control. Exemplary images of Alizarin red staining in 24 well are displayed. Data are shown as box plots with the mean \pm SEM and individual data points. Wilcoxon signed 
Patients with fracture healing disorders often require further surgeries, experience substantial pain

331 and suffer from prolonged functional impairments. While fracture healing is usually completed within 3 to

3324 months, non-unions are identified if healing does not succeed after 9 months (no radiological bridging of

333 fragments is visible; $(1,2)$. Current treatment modalities of non-union include surgical revision, autologous

334 bone grafting or local stimulation of healing by e.g. rhBMP-2 for local delivery into the fracture gap.

335 However, several adverse effects have been reported with BMP-2 and thus clinical usage is strongly

336 restricted (63). Preventive measures for patients potentially at risk do not yet exist. Therefore, strategies that

337 would allow to treat patients with a probable lack in their healing capability early on would be highly

338 desirable. Our single-center retrospective study identified specifically patients with dysregulation of the

339 immune system (e.g. RA) or disturbed capacities in angiogenesis (e.g. smoking) as being at high risk to

340 experience a non-union (Table 1), which is in agreement with other reports $(6,64)$. Induction of regenerative

341 processes within the initial phase of fracture healing depends on an adequate cellular adaptation to the

342 hypoxic microenvironment of the fracture gap (31). Therefore, we hypothesized that stabilization of HIF-

$3431 \alpha$ could be an effective approach for the prevention of fracture healing disorders. Based on a systematic

344 literature review, we identified DFO as stimulatory factor in bone regeneration for both a variety of clinical

345 bone healing settings (including mandibular defects and critical size defects) and across various species and

346 models (mouse, rat, rabbit) (Table 2). In vitro, we could demonstrate the effect of a single-dose application

347 of DFO alone and DFO in synergy with MIF to counteract a GC-induced inhibition of calcification during

348 osteogenic differentiation of hMSCs. Finally, we could demonstrate in an in vivo proof-of-concept

349 experiment that DFO alone or in synergy with MIF can prevent delayed bone healing in a locally impaired

350 healing model that uses a bovine Col I scaffold in a mouse-osteotomy.

351 Since GCs negatively affect bone metabolism, it is not surprising that GCs have also been reported

352 to negatively affect bone healing (65). Although GCs are essential for osteogenic differentiation at low

353 concentrations, high GC concentrations inhibit osteogenic differentiation and proliferation while favoring

354 adipogenesis (66-71). Therefore, we selected the potent GC dexamethasone to effectively inhibit in vitro 
calcification of hMSCs as an in vitro model for disturbed bone synthesis to test the ability of HIF-stabilizers

to re-establish osteogenic differentiation within the present study (Fig. 1). Of note, beside its stabilizing osteogenic differentiation as shown in the present study, has not been reported, yet.

However, the ability of MIF, the natural counter-regulator of GC action, to support hypoxia-mediated

HIF-1 $\alpha$ stabilization has been shown independently by two groups $(33,74)$. The in vitro findings presented

here demonstrate that the impact of MIF alone is not sufficient to overcome the high-dose GC-mediated suppression of osteogenic differentiation. However, MIF - in combination with DFO - synergistically

enhanced the counteracting potential of DFO on hMSC calcification (Fig. 1). Taken together, DFO and its combination with MIF re-established osteogenic-induced hMSC calcification in a high-dose GC in vitro model for disturbed bone synthesis.

When analyzing HIF stabilizers/enhancers in an in vivo model of delayed healing using a mouseosteotomy-model, we clearly demonstrated that application of DFO alone or in combination with MIF enhanced mineralized callus formation after 14 and 21 days (Fig. 2). This is in line with several previous reports on DFO administered in rat- or mouse-osteotomy-models of Ossa irregularia (mandibula or zygomatic arch) and long bones (femur or tibia) $(35-48,50,53,54,56,75,76)$. All studies showed a strong positive effect of DFO on bone and vessel formation although the application routes and concentrations differed. Most comparable to our present work are the studies of Wan et al. and Yao et al. using the mouseosteotomy-model with medullary pin fixation, but with day-wise repeated local injections of $200 \mu \mathrm{M}$ DFO $(35,77)$. In the study presented here, our hypothesis was that a single dose of DFO alone or in combination with MIF is sufficient to accelerate bone formation. Indeed, we could verify our hypothesis by providing evidence for the enhanced mineralized bone formation at later time points (14 and 21 days; Fig. 2). Although mineralization and bone formation, we assume that the DFO effect alone is strong enough and, therefore, 
masks the potential additional effect of MIF. However, comparing the histomorphometric results on mineralized bone formation endosteal or intracortical, MIF alone and in combination with DFO showed significantly more mineralized bone in the endosteal compartment after 14 days (Fig. 2) indicating a pivotal role for MIF alone during fracture healing. Ondara et al. described higher expression levels of MIF during the fracture healing process, which has been also described in other regenerative processes such as wound healing $(78,79)$. MIF deficient mice showed impaired fracture healing caused by a reduced number of osteoclasts and increased osteoid production (80). In our hands, MIF, DFO and MIF/DFO strongly enhanced revascularization much faster than in the control group as shown by the ingrowth of $\mathrm{CD} 31^{\text {hi }} \mathrm{Emcn}{ }^{\text {hi }}$ ECs into the osteotomy gap (week 2 vs. 3; Fig. 3). Kusumbe et al. described these cells to be part of a bone tissue specific vessel subtype linking angiogenesis and bone formation via Notch and HIF-1 $\alpha$ signaling and located the $\mathrm{CD} 31^{\text {hi }} \mathrm{Emcn}^{\text {hi }} \mathrm{ECs}$ to the bone surfaces and into the growth plate $(81,82)$. Moreover, stabilizing HIF by hypoxia or DFO leads to an induction of VEGF expression in different cell types being the major driver of vascularization also during fracture healing $(37,52,76)$.

In addition, we observed an accelerated recruitment of macrophages and osteoclast activity based on TRAP activity in the DFO and MIF/DFO group (Fig. 4). DFO strongly supported resorption of biomaterial by enhancing osteoclast activity (75), while MIF is well-known to promote osteoclastogenesis by interacting with the RANKL pathway (80, 83-85). Furthermore, macrophages play a pivotal role during the whole fracture healing process. Most importantly they promote vascularization and angiogenesis by degrading ECM, which supports the release of angiogenic factors $(86,87)$. Very recently, we have demonstrated the close interconnection between macrophages and vessel formation during fracture healing (57). process. The advantage of synthetic, biodegradable hydrogels such as dPG is the possibility to adjust the properties of the hydrogel to the specific requirements of the fracture gap. We found that the combination of DFO with dPG could be a promising approach (Fig. 5). However, further studies are needed to optimize the delivery system by further modifications (88). Until today, no appropriate delivery system has been approved clinically. 
In summary, our data provides convinving evidence on the potential of DFO to accelerate bone healing by enhancing mineralization and vessel formation. In addition, MIF alone used at a concentration of $100 \mathrm{ng} / \mathrm{ml}$ rather showed inhibitory properties in the regeneration process. The additional effect of MIF on top of the DFO effect was only seen for a few outcomes - e.g. vessel formation. Therefore, it can be supposed that MIF acts concentration-dependent, and further studies on the dosage finding are needed. Here,

412 we showed that the combination of HIF-stabilizers can counteract delayed fracture healing. DFO is

413 approved by the FDA, commercially available (e.g. Desferal® by Novartis AG) and listed on World Health

414 Organization's List of Essential Medicines. We consider DFO as suitable for rapid clinical translation to

415 improve fracture healing and to be used as preventive strategy to avoid bone healing disorders in patients at

416 high risk (e.g. RA and smoking). Therefore, we are currently striving to start a multi-centric confirmatory

417 study with the long-term goal of clinical translation.

\section{$418 \quad$ Limitations}

In the present study, hMSCs were isolated via migration from the bone marrow although normal protocols recommend density gradient centrifugation. We see increased cell numbers that can be an

421 indication for higher heterogeneity in the following cell culture, which can influence the experimental 422 outcomes. Density gradient centrifugation also has disadvantages such as the loss of smaller cell populations 423 including high proliferative hMSCs. Moreover, there are varying protocols for density gradient 424 centrifugation provided in the literature which does not guarantee reproducibility (89). Furthermore, there 425 is strong evidence in the literature that freshly isolated hMSCs differ from isolated and cultivated hMSCs 426 in their transcriptome and secretome indicating that conclusion from in vitro studies should be translated 427 carefully towards in vivo assumptions (90). In the present study, the in vitro studies were rather used as a 428 tool to get insights for further in vivo studies than investigating specific pathways.

For the in vitro studies on DFO/MIF in our GC-induced delayed calcification assay, all hMSCs were

430 expanded and cultivated in monolayer under normoxic condition which does not parallel the normal bone 431 marrow niche, particularly 3D and hypoxia $(91,92)$. Moreover, a heterogenic population of hMSCs was 432 used for the assays while distinct subpopulations can be influenced differently by the treatments (93). In 
433 addition, high dexamethasone concentrations were required to mimic significant inhibitory effects of GCs

434 in vitro. Those concentrations do not resemble clinically used dosages.

435 Additionally, it should be taken into account that the present study was conducted in mice, and the 436 interpolation to the human is limited. In general, in orthopedic research rodents as well as large animal 437 models are most commonly used. Mice are favored for basic research questions due to the possibility of 438 genetical modifications. In contrast, sheep or pigs are preferred for translational approaches, and rats are 439 more often used for pharmacological interventions and toxicological studies. Most animal species show 440 slight analogies to the human bone macro- and microstructure. Main differences between mice and humans 441 comprise permanent opening of the growth plate in the epiphyses of long bones leading to a lifelong skeletal 442 modeling, the lack of a Haversian system and low cancellous bone content at the epiphyses of long bones $443(94,95)$. Here, a mouse-osteotomy-model was used which does not completely heal within a time period of 44421 days (osteotomy gap $0.7 \mathrm{~mm}$ ) in the control group. Thus, an improvement in the healing process can be 445 seen in treated groups. This model only works in female mice since the bone healing process is slower in 446 females than in males (96). However, it is not always possible to determine the exact time frames for every 447 phase during the fracture healing process which makes the interpretation much more complex and might 448 impact especially small differences. This might be a reason why the proposed beneficial effect of MIF is 449 not visible indicating a more technical and methodical challenge rather than a biological non-function. 
In cooperation with the Center for Musculoskeletal Surgery, Charité-Universitätsmedizin Berlin, patient files from patients who were once stationary treated in the hospital during 2012 were screened for ICD-10 classifications M 84.0 (malunion of fracture), M 84.1 (nonunion of fracture) or M 84.2 (delayed union of fracture). Impairment was confirmed by x-rays and patient information as well as patient's history. Ethical approval for the search algorithm and evaluation sheet was provided by the local ethics committee (EA1/349/13). Due to the retrospective character and anonymization, no consent by the included patients was needed. The selected patient files were additionally reviewed by orthopedic experts before inclusion in the study. Therefore, $\mathrm{x}$-rays and patient information as well as history were re-analyzed in detail. Exclusion criteria were age $<18$ years, open fractures and metastases close to the fracture location. Collected data included age, sex, birthday, body height and weight, and fracture related patient's history including cause, treatment location and complications. In addition, information on lifestyle (e.g. alcoholism, smoking), comorbidities and medications were gathered. The BMI was calculated based on body height and weight.

For statistical analysis, the modelling was performed based on univariable and multivariable logistic regression using SPSS V. 22. The first model was built to determine the potential influence of each variable on the fracture healing outcome. The second model served to identify potential confounding factors and verify the independent contribution of variables to the fracture healing outcome.

\section{Systematic literature review}

“bone formation”[Tiab] OR “osteotomy”[Tiab]) - Filters activated: Publication date to 2019/02/28. Google

474 scholar was searched in addition with the terms: deferoxamine, bone healing, fracture. The detailed search

475 strategy is comprehensively explained in figure $\mathbf{S 2}$ following the PRISMA guidelines and recommendations 


\section{Study design - In vitro and in vivo studies}

The overall hypothesis of the study was that the local application of MIF/DFO in long bone fractures enhances new bone formation (osteoinduction) and can be used to accelerate fracture healing for the treatment and prevention of fracture healing disorders. For the in vitro studies, the endpoints were previously defined by hMSC calcification (Alizarin red staining). For the in vivo study the primary endpoint was the

482 bone formation rate (bone volume/total volume) as measured via ex vivo $\mu \mathrm{CT}$ after 2 weeks. Additional 483 endpoints were defined by histomorphometry. Four time points were determined for additional endpoint 484 measurements. The healing outcome was investigated via ex vivo $\mu \mathrm{CT}$ and histology at day 14 and 21 . Two operated animals were excluded due to infection in the osteotomy gap and one animal was partially excluded (only included for ex vivo $\mu \mathrm{CT}$ ) due to an oblique fixation.

In vitro studies using hMSCs were performed as proof of concept and possibility to determine an adequate concentration of DFO/MIF to be used in vivo. hMSCs were used from 4 to 6 different donors (biological replicates) in at least triplicates per experiment and condition (technical replicates). Data was only excluded if donors failed to differentiate as indicated by a positive control that was carried out on every plate. Only hMSCs that passed characterization were used. Calcification was measured via Alizarin red staining as selected prospectively.

For the in vivo study, power analysis was performed prior to animal tests (nQuery) to determine the animal number with the results to use a minimum of 6 animals to attain worthwhile results and was provided in detail with the animal experiment application. All analyses were performed blinded for the experimenter by randomly numbering the animals. Animals were randomized for pairs and treatment groups, although animals in one cage were treated with the same substances.

\section{hMSC cultivation and calcification assay}

Bone marrow was collected from patients undergoing total hip replacement at the Center for

500 Musculoskeletal Surgery, Charité-Universitätsmedizin Berlin. Samples were registered and distributed by 501 the "Tissue Harvesting" Core Facility of the Berlin Institute of Health Center for Regenerative Therapies 502 (BCRT) (table S3). Written consents were gathered from all patients. All protocols were approved by the 
503 local ethics committee (EA1/012/13) and performed according to the Helsinki Declaration. hMSC isolation,

504 expansion and full characterization (FACS, differentiation) was performed as described previously (56, 97).

505 Expansion was done with DMEM plus GlutaMAX (Thermo Fischer Scientific), 10\% FCS (PAA 506 Laboratories), $1 \%$ Penicillin-Streptomycin (Thermo Fischer Scientific) at $37^{\circ} \mathrm{C}$ in $5 \% \mathrm{CO}_{2}$ atmosphere (app.

$50718 \% \mathrm{O}_{2}$ ). Cells were used within passage 4-7. For the calcification assay, hMSCs were transferred to a 96-

508 well plate with a density of $1 \times 10^{4}$ cells/well, cultivated for $24 \mathrm{~h}$ and treated with osteogenic medium (OM)

509 consisting of DMEM, 10\% FCS, 1\% Penicillin-Streptomycin, $10 \mathrm{mM} \beta$-glycerophosphate, $10^{-8} \mathrm{M}$

510 dexamethasone water-soluble and 0.1 mM L-ascorbic acid-2-phosphate (Sigma Aldrich). Dexamethasone,

511 Deferoxamine mesylate salt (DFO; Sigma Aldrich) and MIF (lab own production) were supplemented in

512 different concentrations to the medium. Medium was changed weekly. For the release studies OsteoDiff

513 (Miltenyi Biotech) was used supplemented with 1\% Penicillin-Streptomycin. For Alizarin red staining cells

514 were fixed with $4 \%$ formaldehyde (15 min RT; Carl Roth), washed twice with PBS and stained with $0.5 \%$

515 Alizarin Red (Sigma Aldrich) in $\mathrm{H}_{2} \mathrm{O}_{\text {dest }}(\mathrm{pH} 4)$ for 10 min at RT followed by 4 washing steps with $\mathrm{H}_{2} \mathrm{O}_{\text {dest }}$

516 and application of $10 \%$ cetylpyridinium chloride solution (AppliChem) for $30 \mathrm{~min}$ at RT. Supernatants were

517 transferred to a new 96 well plate and measured with a Synergy HT plate reader (BioTek Instruments) at a

518 wavelength of $562 \mathrm{~nm}$ (reference wavelength $630 \mathrm{~nm}$ ) for quantification.

\section{Animals, housing and osteotomy}

Female C57BL/6N mice (12 weeks; body weight 20 - 25 g; Charles River Laboratories) were housed

521 in the Charité animal facility (FEM; semi-sterile - outside the SPF barrier) in pairs in Euro standard Type II

522 clear-transparent plastic cages and kept under obligatory hygiene standards monitored according to the

523 FELASA standards. Nesting material was provided in sufficient amount while pipes and houses were

524 withdrawn after osteotomy to avoid possible entanglement with the used external fixator. Food and water

525 were available ad libitum and the temperature was $\left(20 \pm 2{ }^{\circ} \mathrm{C}\right)$ controlled with a $12 \mathrm{~h} \mathrm{light/dark}$ period and

526 a humidity of $45-50 \%$. 
All experiments were carried out with ethical permission according to the policies and principles established by the Animal Welfare Act, the National Institutes of Health Guide for Care and Use of approved by the local legal representative animal rights protection authorities (Landesamt für Gesundheit und Soziales Berlin: G 0111/13, 0039/16). Pain management and osteotomy were performed as described in detail previously $(56,57,98,99)$. In short, for analgesia a buprenorphine injection $(0.1 \mathrm{mg} / \mathrm{kg}$; Temgesic,

RB Pharmaceuticals Limited) s.c. was given prior to the surgery and Tramadol $(0.1 \mathrm{mg} / \mathrm{ml}$; Drops,

Grünenthal $\mathrm{GmbH}$ ) was applied with the drinking water for the first 3 post-operative days. Anesthesia was

conducted with isoflurane and $\mathrm{O}_{2}$ supplementation and mice were prepared with eye ointment (Bayer

Pharma AG) and clindamycin s.c. (0.02 ml; Ratiopharm $\mathrm{GmbH})$. Osteotomy with an external fixator

(MouseExFix, RISystem) was performed at the left femur creating a $0.70 \mathrm{~mm}$ osteotomy gap with a Gigli

wire saw. The osteotomy gap was filled with PBS-soaked ACS (control; Lyostypt, B. Braun) or MIF and/or

DFO solved in PBS applied on the ACS (treatment groups; $100 \mathrm{ng} / \mathrm{ml}$ and/or $250 \mu \mathrm{M}$, respectively) (56,

540 98). After skin closure, mice received warmed $\mathrm{NaCl}(0.2 \mathrm{ml})$ s.c returned to their cages with a prepared nest

541 and an infrared radiator. Animals were euthanized via cervical dislocation after 3, 7, 14 and 21 days in deep

542 anesthesia (no deep pain perception) after intracardial blood collection. Osteotomized femora were collected

543 and either fixed with 4\% paraformaldehyde (PFA; Electron Microscopy Sciences) for 6-8 $\mathrm{h}$ at $4{ }^{\circ} \mathrm{C}$.

\section{Ex vivo micro computed tomography $(\mu C T)$}

PFA-fixed femora were treated with an ascending sucrose solution $(10 \%, 20 \%, 30 \%)$ for $24 \mathrm{~h}$,

respectively at $4^{\circ} \mathrm{C}$. Scanning of 191 slices was performed after removal of the pins and external fixator

547 with an isotropic voxel size of $10.5 \mu \mathrm{m}(70 \mathrm{KVp}, 114 \mu \mathrm{A}$; SCANCO $\mu \mathrm{CT}$ Viva 40), aligned scan axis along

548 the diaphyseal axis of the femora and 3D reconstruction and analyses were performed using the provided

549 software package as described previously and applying a fixed global threshold of $240 \mathrm{mg} \mathrm{HA} / \mathrm{cm}^{3}$ for the

550 automatic 3D callus tissue analysis $(56,98)$. Nomenclature and analysis were conducted in accordance with

551 published recommendations (100). 


\section{Histology and immunofluorescence}

After $\mu \mathrm{CT}$ scanning, femora were cryo-embedded without decalcification according to the Kawamoto

554 et al. method (101). For Movat's pentachrome staining slices $(7 \mu \mathrm{m})$ were air dried for $30 \mathrm{~min}$, fixed with

$5554 \%$ PFA for 10 min and washed with $\mathrm{H}_{2} \mathrm{O}_{\text {dest }}$ for $5 \mathrm{~min}$. The staining procedure was conducted using a

556 protocol already been published $(56,102)$. The staining results allowed to distinguish between different

557 tissues: mineralized bone or mineralized cartilage appear yellow, hyaline cartilage green, cytoplasm reddish,

558 cell nuclei blue-black and the surrounding muscles are colored in reddish. When combined with Von Kossa

559 staining - the following staining steps were conducted before the Movat's pentachrome staining: 3\% (w/v)

560 silver nitrate solution (10 min), washing step with $\mathrm{H}_{2} \mathrm{O}_{\text {dest }}$, sodium carbonate formaldehyde solution (2 min),

561 washing step with tap water, $5 \%$ (w/v) sodium thiosulphate solution (5 min), washing step with tap water

562 and $\mathrm{H}_{2} \mathrm{O}_{\text {dest. }}$ Images were taken with a light microscope (Leica) in a 2.5x magnification and the program

563 Axiovision (Carl Zeiss Microscopy). The Acid Phosphatase, Leukocyte (TRAP) Kit (Sigma Aldrich) was

564 used to stain for TRAP. Manufacturer's instructions were followed, and pictures were taken with a light

565 microscope (Leica).

566 For immunofluorescence staining the following primary antibodies: CD31/PECAM-1 (goat 567 polyclonal unconjugated, AF2628, R\&D Systems, 1:100), Emcn (V.7C7 unconjugated, sc-65495, 1:100),

568 F4/80 (Cl:A3-1 unconjugated, MCA497G, 1:400), Osx (rabbit polyclonal, sc-22536-R, 1:200) and 569 secondary antibodies (all Thermo Fischer Scientific; 1:500): anti-rat conjugated AF594 (A21209), anti570 rabbit conjugated AF488 (A21206), anti-rat conjugated AF647 (A21247), anti-goat conjugated AF647 571 (A21447) or anti-goat A568 (A11057) were used. Staining procedure was performed in a wet section as 572 published earlier $(56,57)$. Pictures were taken with a fluorescence microscope BZ 9000 (Keyence). Image 573 analysis was performed with ImageJ $(56,57,98)$.

\section{DFO release assay}

575 In order to measure the DFO concentration in the supernatant we established the method described 576 by Fielding and Brunström 1964 (103). The method is based on the ability of DFO to bind iron and therefore 
577 reduces ferric chloride $\left(\mathrm{Fe}^{3+}\right)$ to ferrioxamine (red-brown compound). Measurements were taken including

578 all components (citric acid and $\mathrm{Na}_{2} \mathrm{HPO}_{4}$ ) and a ferric chloride concentration of $1.5 \mathrm{mg} / 100 \mathrm{ml}$ at a

579 wavelength of $450 \mathrm{~nm}$ (Synergy HT plate reader, BioTek Instruments). Release kinetic experiments were

580 performed in PBS. Hydrogel formation was performed as described in detail before (104). $100 \mu$ of $20 \%$

$581 \mathrm{dPG}$ or dPGS were mixed with $10.000 \mu \mathrm{M}$ DFO and polymerized for $1 \mathrm{~h}$ at $37^{\circ} \mathrm{C}$. For release kinetic and

582 transfer assay $200 \mu \mathrm{l}$ PBS were added and supernatants were collected every $24 \mathrm{~h}$. For transfer assay to HEK

583293 cells supernatant was collected after $24 \mathrm{~h}$ and mixed with normal expansion medium (1:10). HEK 293

584 were treated for $24 \mathrm{~h}$ before collecting protein and performing HIF- $1 \alpha$ western blot as described before (105).

585 For co-cultivation with hMSCs, hydrogels were polymerized in 24-well transwell inserts (Sarstedt;

586 polyethylene terephthalate membrane with $8 \mu \mathrm{m}$ pore size). After polymerization, hydrogels were treated

587 for $12 \mathrm{~h}$ with osteogenic medium at $37^{\circ} \mathrm{C}$ for equilibration and transferred to a 24 -well plate seeded with

588 hMSCs. Co-cultivation was performed for 14 days with supplementation of osteogenic medium. Alizarin

589 red staining was used to visualize calcification of hMSCs.

\section{$590 \quad$ Statistical analysis}

Statistical analysis was carried out with GraphPad Prism V.8 software. All values from in vitro assays were expressed as the mean \pm SD or SEM when measured in $>$ duplicates and all values from animal experiments are depicted as median \pm ranges (box and whiskers plot with individual data points). Kruskal

594 Wallis test with Dunn's multiple comparison test and Wilcoxon-signed rank test were applied in case of 595 lack of Gaussian distribution that was tested before via Kolmogorov-Smirnov test. A p-value $<0.05$ was 596 considered as statistically significant. In some cases, statistical trends are indicated with a hashtag (\#) when 597 biologically relevant. 


\section{References}

600 1. D. J. Hak, D. Fitzpatrick, J. A. Bishop, J. L. Marsh, S. Tilp, R. Schnettler, H. Simpson, V. Alt, $601 \quad$ Delayed union and nonunions: epidemiology, clinical issues, and financial aspects. Injury 45 Suppl

2. K. D. Hankenson, G. Zmmerman, R. Marcucio, Biological Perspectives of Delayed Fracture Healing. Injury 45, S8-S15 (2014).

3. R. Zura, Z. Xiong, T. Einhorn, J. T. Watson, R. F. Ostrum, M. J. Prayson, G. J. Della Rocca, S. Mehta, T. McKinley, Z. Wang, R. G. Steen, Epidemiology of Fracture Nonunion in 18 Human Bones. JAMA Surg 151, e162775 (2016).

4. A. H. Karladani, H. Granhed, J. Karrholm, J. Styf, The influence of fracture etiology and type on fracture healing: a review of 104 consecutive tibial shaft fractures. Arch Orthop Trauma Surg 121, 325-328 (2001).

5. J. A. Scolaro, M. L. Schenker, S. Yannascoli, K. Baldwin, S. Mehta, J. Ahn, Cigarette smoking increases complications following fracture: a systematic review. J Bone Joint Surg Am 96, 674-681 (2014).

6. L. Claes, S. Recknagel, A. Ignatius, Fracture healing under healthy and inflammatory conditions. Nat Rev Rheumatol 8, 133-143 (2012).

7. B. Dominiak, W. Oxberry, P. Chen, Study on a nonhealing fracture from a patient with systemic lupus erythematosus and its pathogenetic mechanisms. Ultrastruct Pathol 29, 107-120 (2005).

8. B. Stromqvist, Hip fracture in rheumatoid arthritis. Acta Orthop Scand 55, 624-628 (1984).

9. I. Dumic-Cule, M. Peric, L. Kucko, L. Grgurevic, M. Pecina, S. Vukicevic, Bone morphogenetic proteins in fracture repair. International Orthopaedics 42, 2619-2626 (2018).

10. G. S. Krishnakumar, A. Roffi, D. Reale, E. Kon, G. Filardo, Clinical application of bone morphogenetic proteins for bone healing: a systematic review. Int Orthop, (2017).

11. M. C. Simmonds, J. V. Brown, M. K. Heirs, J. P. Higgins, R. J. Mannion, M. A. Rodgers, L. A. Stewart, Safety and effectiveness of recombinant human bone morphogenetic protein-2 for spinal fusion: a meta-analysis of individual-participant data. Ann Intern Med 158, 877-889 (2013).

12. T. A. Einhorn, L. C. Gerstenfeld, Fracture healing: mechanisms and interventions. Nat Rev Rheumatol 11, 45-54 (2015).

13. P. Kolar, K. Schmidt-Bleek, H. Schell, T. Gaber, D. Toben, G. Schmidmaier, C. Perka, F. Buttgereit, G. N. Duda, The early fracture hematoma and its potential role in fracture healing. Tissue Eng Part B Rev 16, 427-434 (2010).

14. C. J. Kowalczewski, S. Tombyln, D. C. Wasnick, M. R. Hughes, M. D. Ellenburg, M. F. Callahan, T. L. Smith, M. E. Van Dyke, L. R. Burnett, J. M. Saul, Reduction of ectopic bone growth in critically-sized rat mandible defects by delivery of rhBMP-2 from kerateine biomaterials. Biomaterials 35, 3220-3228 (2014).

15. K. Schmidt-Bleek, A. Petersen, A. Dienelt, C. Schwarz, G. N. Duda, Initiation and early control of tissue regeneration - bone healing as a model system for tissue regeneration. Expert opinion on biological therapy 14, 247-259 (2014).

16. K. Schmidt-Bleek, H. Schell, J. Lienau, N. Schulz, P. Hoff, M. Pfaff, G. Schmidt, C. Martin, C. Perka, F. Buttgereit, H. D. Volk, G. Duda, Initial immune reaction and angiogenesis in bone healing. J Tissue Eng Regen Med 8, 120-130 (2014).

17. J. Lienau, K. Schmidt-Bleek, A. Peters, F. Haschke, G. N. Duda, C. Perka, H. J. Bail, N. Schutze, F. Jakob, H. Schell, Differential regulation of blood vessel formation between standard and delayed bone healing. J Orthop Res 27, 1133-1140 (2009).

18. D. R. Epari, J. P. Kassi, H. Schell, G. N. Duda, Timely fracture-healing requires optimization of axial fixation stability. J Bone Joint Surg Am 89, 1575-1585 (2007).

19. D. R. Epari, H. Schell, H. J. Bail, G. N. Duda, Instability prolongs the chondral phase during bone healing in sheep. Bone 38, 864-870 (2006).

20. J. Lienau, H. Schell, G. N. Duda, P. Seebeck, S. Muchow, H. J. Bail, Initial vascularization and tissue differentiation are influenced by fixation stability. J Orthop Res 23, 639-645 (2005). 
21. P. Strube, U. Sentuerk, T. Riha, K. Kaspar, M. Mueller, G. Kasper, G. Matziolis, G. N. Duda, C. Perka, Influence of age and mechanical stability on bone defect healing: age reverses mechanical effects. Bone 42, 758-764 (2008).

22. H. Aro, E. Eerola, A. J. Aho, J. Niinikoski, Tissue oxygen tension in externally stabilized tibial fractures in rabbits during normal healing and infection. J Surg Res 37, 202-207 (1984).

23. D. R. Epari, J. Lienau, H. Schell, F. Witt, G. N. Duda, Pressure, oxygen tension and temperature in the periosteal callus during bone healing--an in vivo study in sheep. Bone 43, 734-739 (2008).

24. B. Chen, Y. L. Yan, C. Liu, L. Bo, G. F. Li, H. Wang, Y. J. Xu, Therapeutic effect of deferoxamine on iron overload-induced inhibition of osteogenesis in a zebrafish model. Calcif Tissue Int 94, 353 360 (2014).

25. C. Lu, M. Rollins, H. Hou, H. M. Swartz, H. Hopf, T. Miclau, R. S. Marcucio, Tibial fracture decreases oxygen levels at the site of injury. Iowa Orthop J 28, 14-21 (2008).

26. C. Lu, N. Saless, D. Hu, X. Wang, Z. Xing, H. Hou, B. Williams, H. M. Swartz, C. Colnot, T. Miclau, R. S. Marcucio, Mechanical stability affects angiogenesis during early fracture healing. $J$ Orthop Trauma 25, 494-499 (2011).

27. E. Tøndevold, J. Eriksen, E. Jansen, Observations on long bone medullary pressures in relation to arterial PO2, PCO2 and pH in the anaesthetized dog. Acta Orthop Scand 50, 645-651 (1979).

28. T. Gaber, R. Dziurla, R. Tripmacher, G. R. Burmester, F. Buttgereit, Hypoxia inducible factor (HIF) in rheumatology: low O2! See what HIF can do! Annals of the rheumatic diseases 64, 971-980 (2005).

29. C. Maes, G. Carmeliet, E. Schipani, Hypoxia-driven pathways in bone development, regeneration and disease. Nature Reviews Rheumatology 8, 358 (2012).

30. D. E. Komatsu, M. Hadjiargyrou, Activation of the transcription factor HIF-1 and its target genes, VEGF, HO-1, iNOS, during fracture repair. Bone 34, 680-688 (2004).

31. P. Hoff, T. Gaber, K. Schmidt-Bleek, U. Senturk, C. L. Tran, K. Blankenstein, S. Lutkecosmann, J. Bredahl, H. J. Schuler, P. Simon, G. Wassilew, F. Unterhauser, G. R. Burmester, G. Schmidmaier, C. Perka, G. N. Duda, F. Buttgereit, Immunologically restricted patients exhibit a pronounced inflammation and inadequate response to hypoxia in fracture hematomas. Immunol Res 51, 116-122 (2011).

32. P. Hoff, T. Gaber, C. Strehl, M. Jakstadt, H. Hoff, K. Schmidt-Bleek, A. Lang, E. Rohner, D. Huscher, G. Matziolis, G. R. Burmester, G. Schmidmaier, C. Perka, G. N. Duda, F. Buttgereit, A Pronounced Inflammatory Activity Characterizes the Early Fracture Healing Phase in Immunologically Restricted Patients. Int J Mol Sci 18, DOI: 10.3390/ijms18030583 (2017).

33. T. Gaber, S. Schellmann, K. B. Erekul, M. Fangradt, K. Tykwinska, M. Hahne, P. Maschmeyer, M. Wagegg, C. Stahn, P. Kolar, R. Dziurla, M. Lohning, G. R. Burmester, F. Buttgereit, Macrophage migration inhibitory factor counterregulates dexamethasone-mediated suppression of hypoxiainducible factor-1 alpha function and differentially influences human CD4+ T cell proliferation under hypoxia. J Immunol 186, 764-774 (2011).

34. Y. Wang, C. Wan, L. Deng, X. Liu, X. Cao, S. R. Gilbert, M. L. Bouxsein, M. C. Faugere, R. E. Guldberg, L. C. Gerstenfeld, V. H. Haase, R. S. Johnson, E. Schipani, T. L. Clemens, The hypoxiainducible factor alpha pathway couples angiogenesis to osteogenesis during skeletal development. The Journal of clinical investigation 117, 1616-1626 (2007).

35. C. Wan, S. R. Gilbert, Y. Wang, X. Cao, X. Shen, G. Ramaswamy, K. A. Jacobsen, Z. S. Alaql, A. W. Eberhardt, L. C. Gerstenfeld, T. A. Einhorn, L. Deng, T. L. Clemens, Activation of the hypoxiainducible factor-1alpha pathway accelerates bone regeneration. Proc Natl Acad Sci U S A 105, 686691 (2008).

36. Y. Yan, H. Chen, H. Zhang, C. Guo, K. Yang, K. Chen, R. Cheng, N. Qian, N. Sandler, Y. S. Zhang, H. Shen, J. Qi, W. Cui, L. Deng, Vascularized 3D printed scaffolds for promoting bone regeneration. Biomaterials 190-191, 97-110 (2019). 
37. A. Donneys, A. S. Farberg, C. N. Tchanque-Fossuo, S. S. Deshpande, S. R. Buchman, Deferoxamine enhances the vascular response of bone regeneration in mandibular distraction osteogenesis. Plast Reconstr Surg 129, 850-856 (2012).

38. A. Donneys, D. M. Weiss, S. S. Deshpande, S. Ahsan, C. N. Tchanque-Fossuo, D. Sarhaddi, B. Levi, S. A. Goldstein, S. R. Buchman, Localized deferoxamine injection augments vascularity and improves bony union in pathologic fracture healing after radiotherapy. Bone 52, 318-325 (2013).

39. A. S. Farberg, D. Sarhaddi, A. Donneys, S. S. Deshpande, S. R. Buchman, Deferoxamine enhances bone regeneration in mandibular distraction osteogenesis. Plast Reconstr Surg 133, 666-671 (2014).

40. A. Donneys, S. Ahsan, J. E. Perosky, S. S. Deshpande, C. N. Tchanque-Fossuo, B. Levi, K. M. Kozloff, S. R. Buchman, Deferoxamine restores callus size, mineralization, and mechanical strength in fracture healing after radiotherapy. Plast Reconstr Surg 131, 711e-719e (2013).

41. A. Donneys, S. S. Deshpande, C. N. Tchanque-Fossuo, K. L. Johnson, J. T. Blough, J. E. Perosky, K. M. Kozloff, P. A. Felice, N. S. Nelson, A. S. Farberg, B. Levi, S. R. Buchman, Deferoxamine expedites consolidation during mandibular distraction osteogenesis. Bone 55, 384-390 (2013).

42. A. Donneys, N. S. Nelson, E. E. Page, S. S. Deshpande, P. A. Felice, C. N. Tchanque-Fossuo, J. P. Spiegel, S. R. Buchman, Targeting angiogenesis as a therapeutic means to reinforce osteocyte survival and prevent nonunions in the aftermath of radiotherapy. Head Neck 37, 1261-1267 (2015).

43. A. Donneys, N. S. Nelson, J. E. Perosky, Y. Polyatskaya, J. J. Rodriguez, C. Figueredo, C. A. Vasseli, H. C. Ratliff, S. S. Deshpande, K. M. Kozloff, S. R. Buchman, Prevention of radiationinduced bone pathology through combined pharmacologic cytoprotection and angiogenic stimulation. Bone 84, 245-252 (2016).

44. A. S. Farberg, X. L. Jing, L. A. Monson, A. Donneys, C. N. Tchanque-Fossuo, S. S. Deshpande, S. R. Buchman, Deferoxamine reverses radiation induced hypovascularity during bone regeneration and repair in the murine mandible. Bone 50, 1184-1187 (2012).

45. P. A. Felice, S. Ahsan, A. Donneys, S. S. Deshpande, N. S. Nelson, S. R. Buchman, Deferoxamine administration delivers translational optimization of distraction osteogenesis in the irradiated mandible. Plast Reconstr Surg 132, 542e-548e (2013).

46. K. M. Urlaub, J. V. Lynn, E. G. Carey, N. S. Nelson, Y. Polyatskaya, A. Donneys, A. C. Mazzoli, S. R. Buchman, Histologic Improvements in Irradiated Bone Through Pharmaceutical Intervention in Mandibular Distraction Osteogenesis. J Oral Maxillofac Surg 76, 2660-2668 (2018).

47. S. Guzey, A. Aykan, S. Ozturk, H. Avsever, Y. Karslioglu, A. Ertan, The Effects of Desferroxamine on Bone and Bone Graft Healing in Critical-Size Bone Defects. Ann Plast Surg 77, 560-568 (2016).

48. R. Stewart, J. Goldstein, A. Eberhardt, G. T. Chu, S. Gilbert, Increasing vascularity to improve healing of a segmental defect of the rat femur. J Orthop Trauma 25, 472-476 (2011).

49. B. S. Grewal, B. Keller, P. Weinhold, L. E. Dahners, Evaluating effects of deferoxamine in a rat tibia critical bone defect model. J Orthop 11, 5-9 (2014).

50. T. Matsumoto, S. Sato, Stimulating angiogenesis mitigates the unloading-induced reduction in osteogenesis in early-stage bone repair in rats. Physiological reports 3, (2015).

51. P. Jia, H. Chen, H. Kang, J. Qi, P. Zhao, M. Jiang, L. Guo, Q. Zhou, N. D. Qian, H. B. Zhou, Y. J. $\mathrm{Xu}$, Y. Fan, L. F. Deng, Deferoxamine released from poly(lactic-co-glycolic acid) promotes healing of osteoporotic bone defect via enhanced angiogenesis and osteogenesis. 104, 2515-2527 (2016).

52. X. Shen, C. Wan, G. Ramaswamy, M. Mavalli, Y. Wang, C. L. Duvall, L. F. Deng, R. E. Guldberg, A. Eberhart, T. L. Clemens, S. R. Gilbert, Prolyl hydroxylase inhibitors increase neoangiogenesis and callus formation following femur fracture in mice. J Orthop Res 27, 1298-1305 (2009).

53. W. Zhang, G. Li, R. Deng, L. Deng, S. Qiu, New bone formation in a true bone ceramic scaffold loaded with desferrioxamine in the treatment of segmental bone defect: a preliminary study. $J$ Orthop Sci 17, 289-298 (2012).

54. J. Drager, J. L. Ramirez-GarciaLuna, A. Kumar, U. Gbureck, E. J. Harvey, J. E. Barralet, (*) Hypoxia Biomimicry to Enhance Monetite Bone Defect Repair. Tissue Eng Part A 23, 1372-1381 (2017). 
55. M. Hahne, P. Schumann, M. Mursell, C. Strehl, P. Hoff, F. Buttgereit, T. Gaber, Unraveling the role of hypoxia-inducible factor (HIF)-1alpha and HIF-2alpha in the adaption process of human microvascular endothelial cells (HMEC-1) to hypoxia: Redundant HIF-dependent regulation of macrophage migration inhibitory factor. Microvasc Res 116, 34-44 (2018).

56. A. Lang, M. Kirchner, J. Stefanowski, M. Durst, M. C. Weber, M. Pfeiffenberger, A. Damerau, A. E. Hauser, P. Hoff, G. N. Duda, F. Buttgereit, K. Schmidt-Bleek, T. Gaber, Collagen I-based scaffolds negatively impact fracture healing in a mouse-osteotomy-model although used routinely in research and clinical application. Acta Biomater 86, 171-184 (2019).

57. J. Stefanowski, A. Lang, A. Rauch, L. Aulich, M. Köhler, A. F. Fiedler, F. Buttgereit, K. SchmidtBleek, G. N. Duda, T. Gaber, R. A. Niesner, A. E. Hauser, Spatial Distribution of Macrophages During Callus Formation and Maturation Reveals Close Crosstalk Between Macrophages and Newly Forming Vessels. Frontiers in Immunology 10, (2019).

58. J. How, J. R. Brown, S. Saylor, D. L. Rimm, Macrophage expression of tartrate-resistant acid phosphatase as a prognostic indicator in colon cancer. Histochemistry and cell biology 142, 195204 (2014).

59. C. H. Bucher, C. Schlundt, D. Wulsten, F. A. Sass, S. Wendler, A. Ellinghaus, T. Thiele, R. Seemann, B. M. Willie, H. D. Volk, G. N. Duda, K. Schmidt-Bleek, Experience in the Adaptive Immunity Impacts Bone Homeostasis, Remodeling, and Healing. Front Immunol 10, 797 (2019).

60. K. Licha, P. Welker, M. Weinhart, N. Wegner, S. Kern, S. Reichert, I. Gemeinhardt, C. Weissbach, B. Ebert, R. Haag, M. Schirner, Fluorescence Imaging with Multifunctional Polyglycerol Sulfates: Novel Polymeric near-IR Probes Targeting Inflammation. Bioconjugate Chemistry 22, 2453-2460 (2011).

61. T. Schneider, P. Welker, K. Licha, R. Haag, G. Schulze-Tanzil, Influence of dendritic polyglycerol sulfates on knee osteoarthritis: an experimental study in the rat osteoarthritis model. $B M C$ Musculoskeletal Disorders 16, 387 (2015).

62. H. Türk, R. Haag, S. Alban, Dendritic Polyglycerol Sulfates as New Heparin Analogues and Potent Inhibitors of the Complement System. Bioconjugate Chemistry 15, 162-167 (2004).

63. E. A. Barcak, M. J. Beebe, Bone Morphogenetic Protein: Is There Still a Role in Orthopedic Trauma in 2017? Orthop Clin North Am 48, 301-309 (2017).

64. R. K. Hernandez, T. P. Do, C. W. Critchlow, R. E. Dent, S. S. Jick, Patient-related risk factors for fracture-healing complications in the United Kingdom General Practice Research Database. Acta Orthop 83, 653-660 (2012).

65. Y. Hachemi, A. E. Rapp, A.-K. Picke, G. Weidinger, A. Ignatius, J. Tuckermann, Molecular mechanisms of glucocorticoids on skeleton and bone regeneration after fracture. Journal of molecular endocrinology 61, R75-R90 (2018).

66. I. Carcamo-Orive, A. Gaztelumendi, J. Delgado, N. Tejados, A. Dorronsoro, J. Fernandez-Rueda, D. J. Pennington, C. Trigueros, Regulation of human bone marrow stromal cell proliferation and differentiation capacity by glucocorticoid receptor and AP-1 crosstalk. J Bone Miner Res 25, 2115 2125 (2010).

67. P. L. Chang, H. C. Blair, X. Zhao, Y. W. Chien, D. Chen, A. B. Tilden, Z. Chang, X. Cao, O. M. Faye-Petersen, P. Hicks, Comparison of fetal and adult marrow stromal cells in osteogenesis with and without glucocorticoids. Connect Tissue Res 47, 67-76 (2006).

68. C. D. Hoemann, H. El-Gabalawy, M. D. McKee, In vitro osteogenesis assays: influence of the primary cell source on alkaline phosphatase activity and mineralization. Pathol Biol (Paris) 57, 318-323 (2009).

69. I. H. Song, A. I. Caplan, J. E. Dennis, In vitro dexamethasone pretreatment enhances bone formation of human mesenchymal stem cells in vivo. J Orthop Res 27, 916-921 (2009).

70. G. Tan, P. D. Kang, F. X. Pei, Glucocorticoids affect the metabolism of bone marrow stromal cells and lead to osteonecrosis of the femoral head: a review. Chin Med J (Engl) 125, 134-139 (2012).

71. S. Walsh, G. R. Jordan, C. Jefferiss, K. Stewart, J. N. Beresford, High concentrations of dexamethasone suppress the proliferation but not the differentiation or further maturation of human 


\section{Z.}

72. Z. H. Qu, X. L. Zhang, T. T. Tang, K. R. Dai, Promotion of osteogenesis through beta-catenin signaling by desferrioxamine. Biochem Biophys Res Commun 370, 332-337 (2008).

73. X. Yu, Q. Wan, G. Cheng, X. Cheng, J. Zhang, J. L. Pathak, Z. Li, CoCl2, a mimic of hypoxia, enhances bone marrow mesenchymal stem cells migration and osteogenic differentiation via STAT3 signaling pathway. Cell Biol Int 42, 1321-1329 (2018).

74. M. Winner, A. C. Koong, B. E. Rendon, W. Zundel, R. A. Mitchell, Amplification of tumor hypoxic responses by macrophage migration inhibitory factor-dependent hypoxia-inducible factor stabilization. Cancer Res 67, 186-193 (2007).

75. J. Drager, Z. Sheikh, Y. L. Zhang, E. J. Harvey, J. E. Barralet, Local delivery of iron chelators reduces in vivo remodeling of a calcium phosphate bone graft substitute. Acta Biomater 42, 411419 (2016).

76. B. P. Hertzberg, J. B. Holt, R. D. Graff, S. R. Gilbert, L. E. Dahners, An evaluation of carrier agents for desferoxamine, an up-regulator of vascular endothelial growth factor. J Biomater Appl 27, 10461054 (2013).

77. Q. Yao, Y. Liu, J. Tao, K. M. Baumgarten, H. Sun, Hypoxia-Mimicking Nanofibrous Scaffolds Promote Endogenous Bone Regeneration. ACS Appl Mater Interfaces 8, 32450-32459 (2016).

78. S. Onodera, J. Nishihira, M. Yamazaki, T. Ishibashi, A. Minami, Increased expression of macrophage migration inhibitory factor during fracture healing in rats. Histochem Cell Biol 121, 209-217 (2004).

79. S. C. Gilliver, E. Emmerson, J. Bernhagen, M. J. Hardman, MIF: a key player in cutaneous biology and wound healing. Exp Dermatol 20, 1-6 (2011).

80. T. Kobayashi, S. Onodera, E. Kondo, H. Tohyama, H. Fujiki, A. Yokoyama, K. Yasuda, Impaired fracture healing in macrophage migration inhibitory factor-deficient mice. Osteoporos Int 22, 1955 1965 (2011).

81. A. P. Kusumbe, S. K. Ramasamy, R. H. Adams, Coupling of angiogenesis and osteogenesis by a specific vessel subtype in bone. Nature 507, 323-328 (2014).

82. S. K. Ramasamy, A. P. Kusumbe, L. Wang, R. H. Adams, Endothelial Notch activity promotes angiogenesis and osteogenesis in bone. Nature 507, 376-380 (2014).

83. R. Gu, L. L. Santos, D. Ngo, H. Fan, P. P. Singh, G. Fingerle-Rowson, R. Bucala, J. Xu, J. M. Quinn, E. F. Morand, Macrophage migration inhibitory factor is essential for osteoclastogenic mechanisms in vitro and in vivo mouse model of arthritis. Cytokine 72, 135-145 (2015).

84. S. H. Mun, H. Y. Won, P. Hernandez, H. L. Aguila, S. K. Lee, Deletion of CD74, a putative MIF receptor, in mice enhances osteoclastogenesis and decreases bone mass. J Bone Miner Res 28, 948 959 (2013).

85. L. Zheng, J. Gao, K. Jin, Z. Chen, W. Yu, K. Zhu, W. Huang, F. Liu, L. Mei, C. Lou, D. He, Macrophage migration inhibitory factor (MIF) inhibitor 4-IPP suppresses osteoclast formation and promotes osteoblast differentiation through the inhibition of the NF-kappaB signaling pathway. Faseb j, fj201802364RR (2019).

86. R. P. Pirraco, R. L. Reis, A. P. Marques, Effect of monocytes/macrophages on the early osteogenic differentiation of hBMSCs. J Tissue Eng Regen Med 7, 392-400 (2013).

87. L. J. Raggatt, M. E. Wullschleger, K. A. Alexander, A. C. Wu, S. M. Millard, S. Kaur, M. L. Maugham, L. S. Gregory, R. Steck, A. R. Pettit, Fracture healing via periosteal callus formation requires macrophages for both initiation and progression of early endochondral ossification. Am J Pathol 184, 3192-3204 (2014).

88. X. Bai, M. Gao, S. Syed, J. Zhuang, X. Xu, X.-Q. Zhang, Bioactive hydrogels for bone regeneration. Bioactive materials 3, 401-417 (2018).

89. N. Y. Naung, S. Suttapreyasri, S. Kamolmatyakul, T. Nuntanaranont, Comparative study of different centrifugation protocols for a density gradient separation media in isolation of 
osteoprogenitors from bone marrow aspirate. Journal of oral biology and craniofacial research $\mathbf{4}$, 160-168 (2014).

90. H. Li, R. Ghazanfari, D. Zacharaki, H. C. Lim, S. Scheding, Isolation and characterization of primary bone marrow mesenchymal stromal cells. Ann N Y Acad Sci 1370, 109-118 (2016).

91. A. Papadimitropoulos, E. Piccinini, S. Brachat, A. Braccini, D. Wendt, A. Barbero, C. Jacobi, I. Martin, Expansion of human mesenchymal stromal cells from fresh bone marrow in a 3D scaffoldbased system under direct perfusion. PLoS One 9, e102359 (2014).

92. Y. Zhou, H. Chen, H. Li, Y. Wu, 3D culture increases pluripotent gene expression in mesenchymal stem cells through relaxation of cytoskeleton tension. Journal of cellular and molecular medicine 21, 1073-1084 (2017).

93. H. Oshina, S. Sotome, T. Yoshii, I. Torigoe, Y. Sugata, H. Maehara, E. Marukawa, K. Omura, K. Shinomiya, Effects of continuous dexamethasone treatment on differentiation capabilities of bone marrow-derived mesenchymal cells. Bone 41, 575-583 (2007).

94. P. S. Gomes, M. H. Fernandes, Rodent models in bone-related research: the relevance of calvarial defects in the assessment of bone regeneration strategies. Lab Anim 45, 14-24 (2011).

95. M. Peric, I. Dumic-Cule, D. Grcevic, M. Matijasic, D. Verbanac, R. Paul, L. Grgurevic, V. Trkulja, C. M. Bagi, S. Vukicevic, The rational use of animal models in the evaluation of novel bone regenerative therapies. Bone 70, 73-86 (2015).

96. M. Mehta, H. Schell, C. Schwarz, A. Peters, K. Schmidt-Bleek, A. Ellinghaus, H. J. Bail, G. N. Duda, J. Lienau, A 5-mm femoral defect in female but not in male rats leads to a reproducible atrophic non-union. Arch Orthop Trauma Surg 131, 121-129 (2011).

97. T. Gaber, A. C. K. Brinkman, J. Pienczikowski, K. Diesing, A. Damerau, M. Pfeiffenberger, A. Lang, S. Ohrndorf, G. R. Burmester, F. Buttgereit, P. Hoff, Impact of Janus Kinase Inhibition with Tofacitinib on Fundamental Processes of Bone Healing. Int J Mol Sci 21, (2020).

98. P. Jirkof, M. Durst, R. Klopfleisch, R. Palme, C. Thone-Reineke, F. Buttgereit, K. Schmidt-Bleek, A. Lang, Administration of Tramadol or Buprenorphine via the drinking water for post-operative analgesia in a mouse-osteotomy model. Sci Rep 9, DOI: 10.1038/s41598-41019-47186-41595 (2019).

99. A. Lang, A. Schulz, A. Ellinghaus, K. Schmidt-Bleek, Osteotomy models - the current status on pain scoring and management in small rodents. Lab Anim 50, 433-441 (2016).

100. M. L. Bouxsein, S. K. Boyd, B. A. Christiansen, R. E. Guldberg, K. J. Jepsen, R. Muller, Guidelines for assessment of bone microstructure in rodents using micro-computed tomography. J Bone Miner Res 25, 1468-1486 (2010).

101. T. Kawamoto, K. Kawamoto, Preparation of thin frozen sections from nonfixed and undecalcified hard tissues using Kawamot's film method (2012). Methods Mol Biol 1130, 149-164 (2014).

102. C. Schlundt, T. El Khassawna, A. Serra, A. Dienelt, S. Wendler, H. Schell, N. van Rooijen, A. Radbruch, R. Lucius, S. Hartmann, G. N. Duda, K. Schmidt-Bleek, Macrophages in bone fracture healing: Their essential role in endochondral ossification. Bone 106, 78-89 (2015).

103. J. Fielding, M. Brunstroem, ESTIMATION OF FERRIOXAMINE AND DESFERRIOXAMINE IN URINE. J Clin Pathol 17, 395-398 (1964).

104. S. Hemmati-Sadeghi, P. Dey, J. Ringe, R. Haag, M. Sittinger, T. Dehne, Biomimetic sulfated polyethylene glycol hydrogel inhibits proteoglycan loss and tumor necrosis factor- $\alpha$-induced expression pattern in an osteoarthritis in vitro model. Journal of Biomedical Materials Research Part B: Applied Biomaterials 107, 490-500 (2019).

105. M. Wagegg, T. Gaber, F. L. Lohanatha, M. Hahne, C. Strehl, M. Fangradt, C. L. Tran, K. Schonbeck, P. Hoff, A. Ode, C. Perka, G. N. Duda, F. Buttgereit, Hypoxia promotes osteogenesis but suppresses adipogenesis of human mesenchymal stromal cells in a hypoxia-inducible factor-1 dependent manner. PLoS One 7, e46483 (2012). 
Acknowledgments: The authors would like to thank Gabriela May, Manuela Jakstadt, Gabriela Korus, Marie-Christin Weber and Mario Thiele for excellent technical assistance. Additionally, we like to acknowledge Peter Schlattmann (University of Jena, Germany) for supporting logistic regression analysis. Moreover, Anastasia Rakow and Paul Simon served as orthopedic expert team to re-analyze the cases included in the retrospective study. FACS analyses were performed together with the Core Facility at the

904 German Rheumatism Research Centre. Bone-marrow was provided from the "Tissue Harvesting" Core

905 Facility of the BCRT. This study is a part of Annemarie Lang's PhD thesis as published under

906 https://refubium.fu-berlin.de/handle/fub188/26365. The retrospective study was performed within the

907 doctoral dissertation of Sara Fügener (now Helfmeier) - https://refubium.fu-

908 berlin.de/handle/fub188/3611?show=full. Funding: This study was financially supported by the Berlin

909 Brandenburg Center for Regenerative Therapies (BCRT) and the Berlin Brandenburg School for

910 Regenerative Therapies (BSRT). The work of Timo Gaber was funded by the Deutsche

911 Forschungsgemeinschaft (DFG; no. 353142848). Some data were taken during a refinement study on the

912 pain management in the mouse osteotomy model that was financed by the federal ministry for risk

913 assessment (BfR and Bf3R) in Berlin ("RefineMOMo"; 1328-542). The work of Anja E. Hauser was funded

914 by DFG FOR2165 (HA5354/6-2 to A.E.H.). Author contributions: : K.S.B., G.N.D., M.L., T.G. and F.B.

915 conceived and supervised the research. S.H., A.L., P.H., C.P. and F.B. designed the retrospective study, 916 performed data collection and quality control and performed analysis. A.L., K.S.B. an T.G. designed and 917 performed in vitro and animal experiments. A.L., J.S., A.K., V.S., A.W. A.D. and M.P. performed analysis. 918 A.L., T.G., J.R., S.H.-S., R.H. planned, supervised or performed release assays. A.L., F.B., T.G. and K.S.B. 919 wrote the paper. A.E.H., G.N.D. and M. L. revised manuscript. Competing interests: Authors confirm no 920 competing interest. Data and materials availability: All data associated with this study are present in the 921 paper or the Supplementary Materials. 


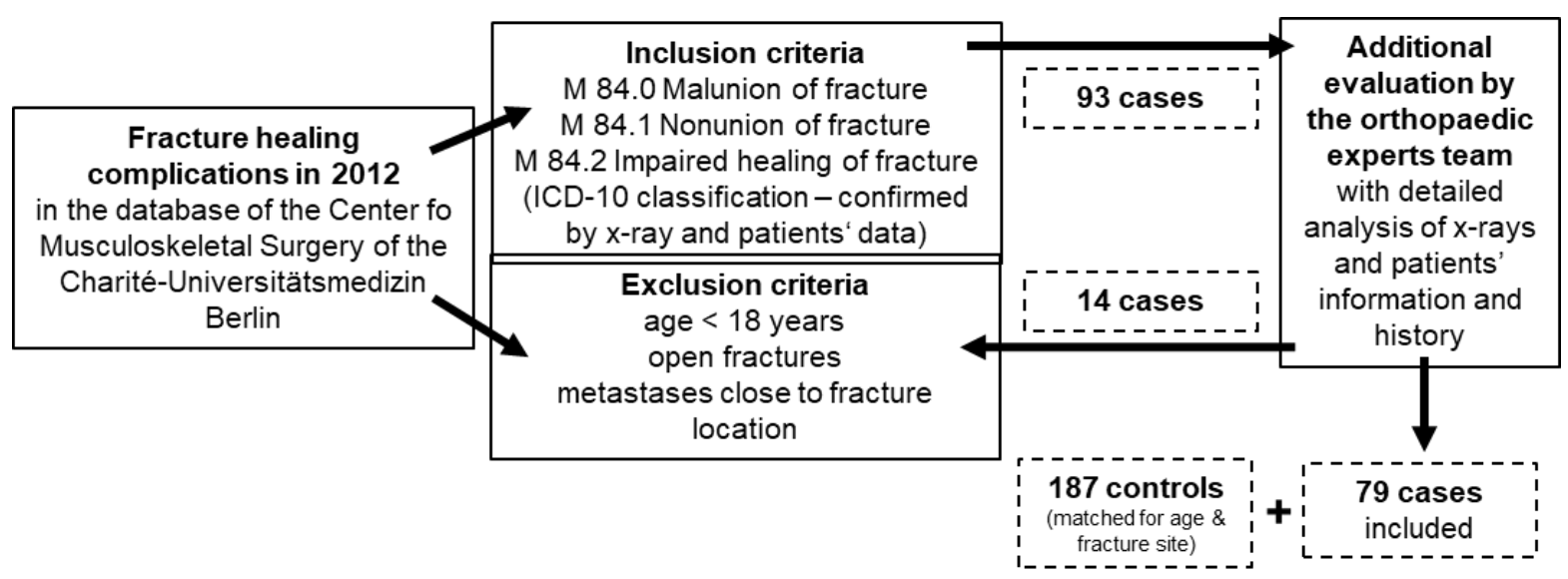

929 Figure S6: Search strategy of the retrospective study including inclusion and exclusion criteria.

930

Table S1: Detailed characterization of the 79 included cases.

\section{Total number in \%}

ICD-10 classification

M 84.0

M 84.1

M 84.2

Fracture localisation

Humerus

Radius, ulna

20

Tibia, fibula

12

61

15.2

Femur

Clavicle

Scaphoid

Vertebrae

Os sacrum

Patella

Metatarsal
11

6

77.2

7.6

3.8 
933 Table S2: Descriptive analysis.

\begin{tabular}{|c|c|c|}
\hline & Controls in \% & Cases in \% \\
\hline \multicolumn{3}{|l|}{ Age } \\
\hline $18-39$ & 26.2 & 30.4 \\
\hline $40-59$ & 36.9 & 34.2 \\
\hline$>60$ & 36.9 & 35.4 \\
\hline \multicolumn{3}{|l|}{ Gender } \\
\hline male & 44.9 & 53.2 \\
\hline female & 55.1 & 46.8 \\
\hline \multicolumn{3}{|l|}{ BMI } \\
\hline$<20$ & 9.9 & 6.5 \\
\hline $20-25$ & 40.7 & 41.6 \\
\hline$>25$ & 49.4 & 51.9 \\
\hline \multicolumn{3}{|l|}{ Alcohol abuse } \\
\hline no & 85.6 & 97.3 \\
\hline yes & 14.4 & 2.7 \\
\hline \multicolumn{3}{|l|}{ Smoking } \\
\hline no & 71.9 & 62.2 \\
\hline yes & 28.1 & 37.8 \\
\hline \multicolumn{3}{|c|}{ Glucocorticoids } \\
\hline no & 97.3 & 93.7 \\
\hline continuously & 1.6 & 6.3 \\
\hline temporarly & 1.1 & 0 \\
\hline \multicolumn{3}{|l|}{ NSAIDs } \\
\hline no & 84.5 & 86.1 \\
\hline continuously & 2.7 & 7.6 \\
\hline ASS-100 mg & 12.8 & 6.3 \\
\hline \multicolumn{3}{|c|}{ Rheumatoid Arthritis } \\
\hline no & 99.5 & 93.7 \\
\hline yes & 0.5 & 6.3 \\
\hline \multicolumn{3}{|l|}{ Osteoporosis } \\
\hline no & 90.8 & 89.9 \\
\hline yes & 9.5 & 10.1 \\
\hline \multicolumn{3}{|c|}{ Arterial Hypertension } \\
\hline no & 66.5 & 57.0 \\
\hline yes & 33.5 & 43.0 \\
\hline \multicolumn{3}{|c|}{ Diabetes Type 2} \\
\hline no & 88.1 & 88.6 \\
\hline yes & 11.9 & 11.4 \\
\hline
\end{tabular}




\begin{tabular}{|c|c|}
\hline \multicolumn{2}{|c|}{$\begin{array}{c}\text { Does local application of Deferoxamine in the fracture gap enhance bone formation }(\mu C T \text {; } \\
\text { histomorphometry) during fracture healing in animal models with normal or disturbed } \\
\text { fractures of long-bones or Ossa irregularia? }\end{array}$} \\
\hline \multicolumn{2}{|c|}{ PICO items } \\
\hline \multicolumn{2}{|c|}{$\begin{array}{l}\text { The disease/health problem of interest: Fracture healing and disorders } \\
\text { The population/species studies: All animal models used for osteotomy; no calvarial defects } \\
\text { The intervention/exposure: Local application of Deferoxamine in the fracture gap } \\
\text { The control population: Placebo, untreated } \\
\text { The outcome measures: } \mu C T \text {; histomorphometry - improvement of fracture healing }\end{array}$} \\
\hline \multirow{4}{*}{$\begin{array}{l}\text { Inclusion criteria } \\
\text { Type of study: Animal studies with controls } \\
\text { Type of animals/population: All animal } \\
\text { models for fracture healing } \\
\text { Type of intervention: Local application of } \\
\text { DFO (no restriction for dosage, timing or } \\
\text { frequency) with or without scaffold }\end{array}$} & Exclusion criteria \\
\hline & Type of study: Non-interventional studies, \\
\hline & $\begin{array}{l}\text { no control group } \\
\text { Type of animals/population: In vitro }\end{array}$ \\
\hline & $\begin{array}{l}\text { studies or human studies } \\
\text { Type of intervention: Combination with } \\
\text { other substances only }\end{array}$ \\
\hline $\begin{array}{l}\text { Outcome measures: } \mu C T \text {, histology, } \\
\text { histomorphometry }\end{array}$ & $\begin{array}{l}\text { Outcome measures: No relevant } \\
\text { outcomes assessed, only x-rays }\end{array}$ \\
\hline Language restriction: English & Language restrictions: Other languages \\
\hline $\begin{array}{l}\text { Publication date restrictions: Articles } \\
\text { published up to Feb } 2019\end{array}$ & $\begin{array}{l}\text { Publication date restrictions: Past date } \\
\text { Type of publication: Reviews or }\end{array}$ \\
\hline $\begin{array}{l}\text { Type of publication: Peer-reviewed, } \\
\text { original, full-text publications }\end{array}$ & non-original papers \\
\hline
\end{tabular}

939 Figure S7: Search strategy of the systematic literature review in accordance with the PRISMA 940 guidelines and recommendations from Syrf and Syrcle. 


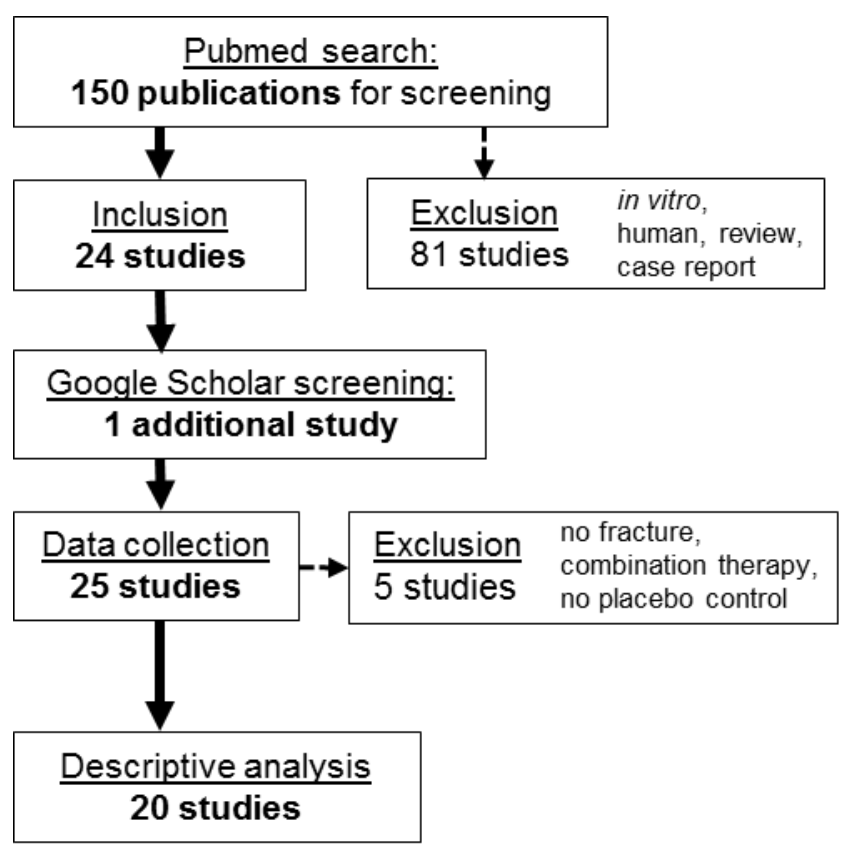

$943 \quad$ Figure S8: Flow diagram of the systematic literature review resulting in the inclusion of 20 studies.

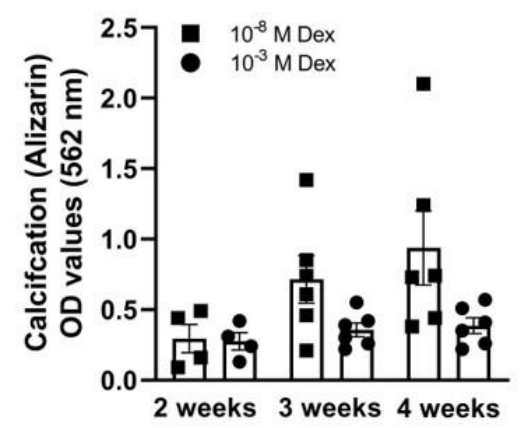

948 Figure S9: In vitro model pre-testing. Determination of the conditions and testing period of the 949 calcification assay for further titration experiments under normoxic conditions ( $N=4-6$; > triplicates). Bar 950 graphs show mean \pm SEM and individual data points. 

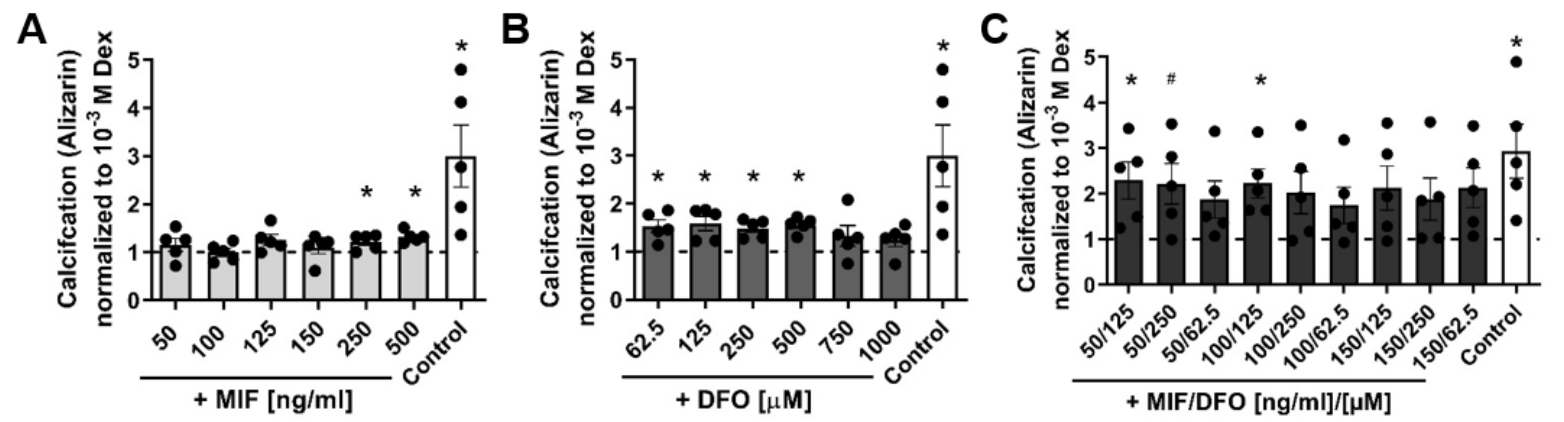

953 Figure S10: Titration of different MIF and DFO concentrations alone $(A, B)$ and in combination $(C)$. hMSCs were cultivated under normoxia for 4 weeks with addition of osteogenic medium (OM). OD values gained after Alizarin red staining were normalized to Dex $10^{-3} \mathrm{M}(\mathrm{N}=4-6$; > triplicates $)$. Bar graphs show mean \pm SEM and individual data points. One sample t-test was used to determine statistical significance towards the hypothetical value of 1. p-values are indicated with ${ }^{\#} P<0.07 ; * P<0.05$. 

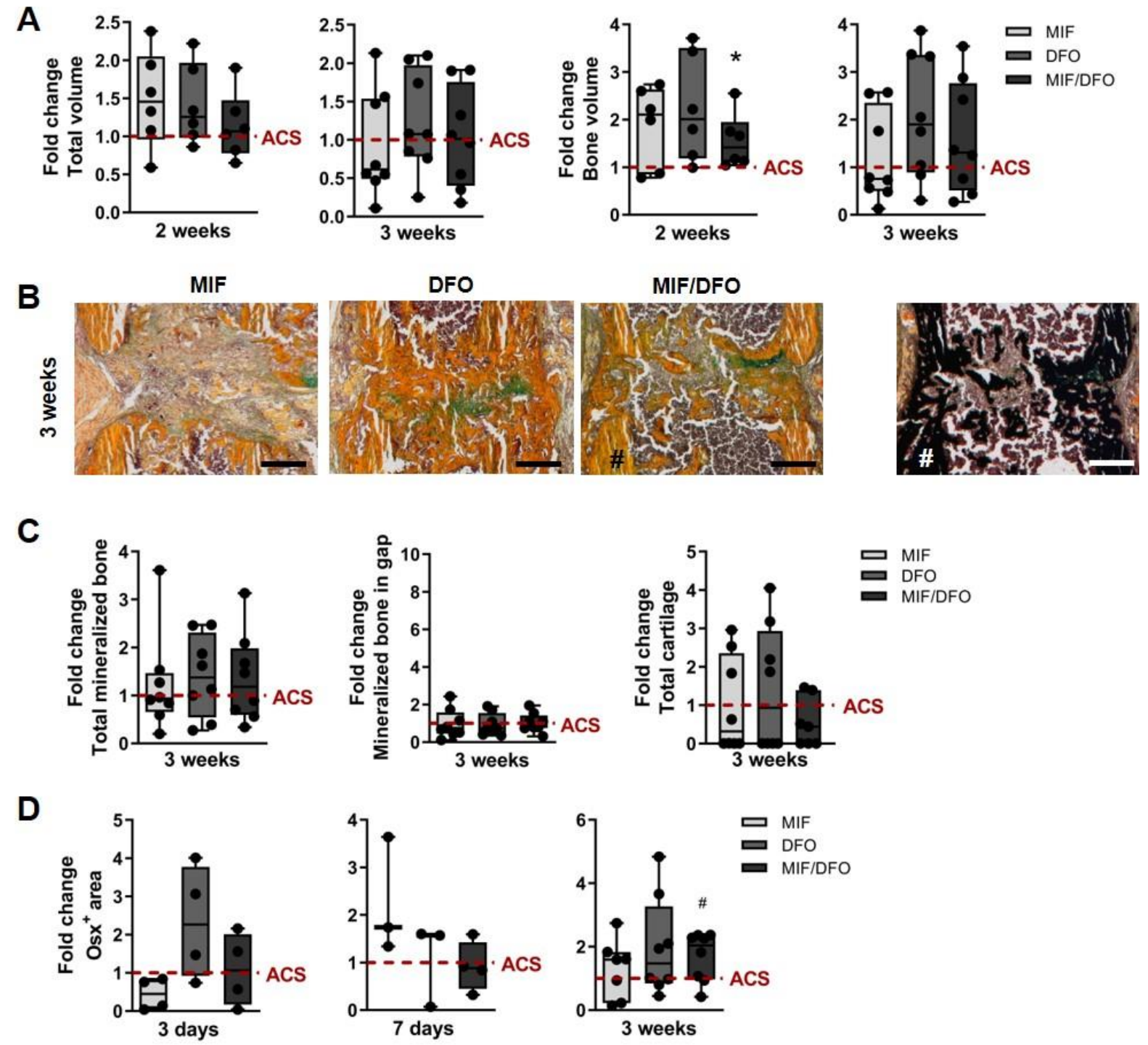

959 Figure S11: Bone regeneration in a delayed healing model after single dose of MIF or/and DFO additional data. (A) MicroCT quantification of total volume and bone volume at 2 weeks and at 3 weeks post-osteotomy normalized to the median of the ACS group (indicated as dotted line $=1$ ). (B) Representative images of Movat's pentachrome staining for each treatment group. yellow-mineralized bone/scaffold; green - cartilage; magenta - bone marrow. Representative images for ${ }^{\#} M I F / D F O, 3$ weeks of von Kossa combined with Movat's pentachrome staining to show the distinction between mineralized bone and residual scaffold. (C) Histomorphometry of Movat's pentachrome staining using ImageJ. Data were normalized to the median of the ACS group (indicated as dotted line =1). (D) Quantification of Osx staining at 3, 7 days and 3 weeks. Data are shown as box plots with the median as horizontal line, interquartile range as boxes, minimum/maximum as whiskers and individual data points. Wilcoxon signed rank test was applied to determine difference against the ACS control group (hypothetical value $=1$ ) and Kruskal Wallis test with Dunn's multiple comparison test was used to compare groups. ${ }^{\#} P<0.07 ; * P<0.05$. 


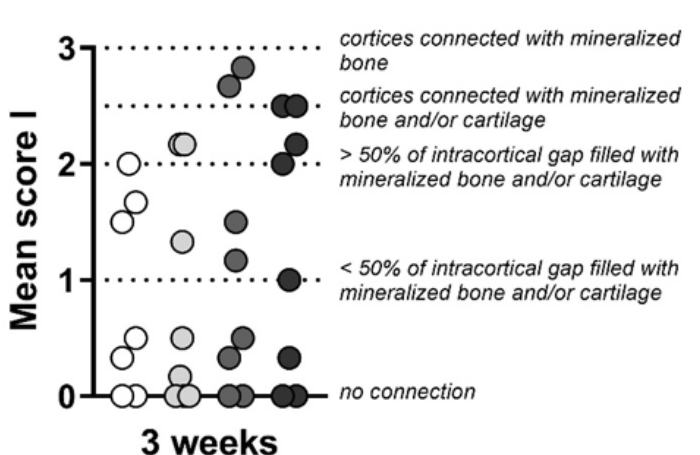

3 weeks

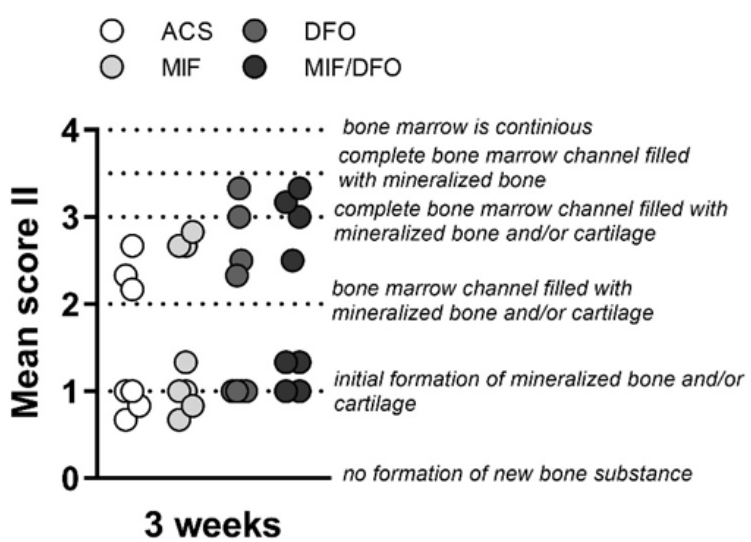

$\bigcirc$ ACS $\bigcirc$ DFO

3 weeks
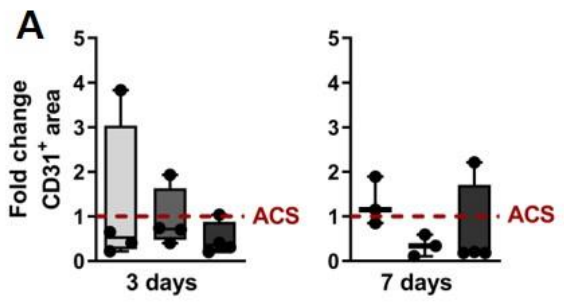

B
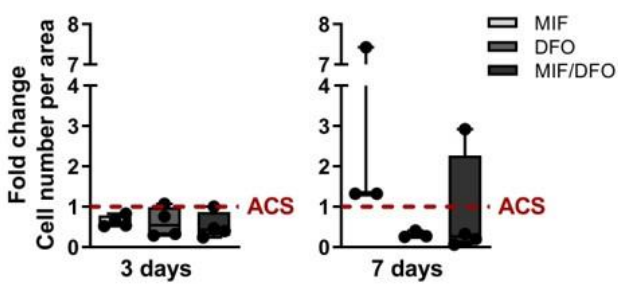
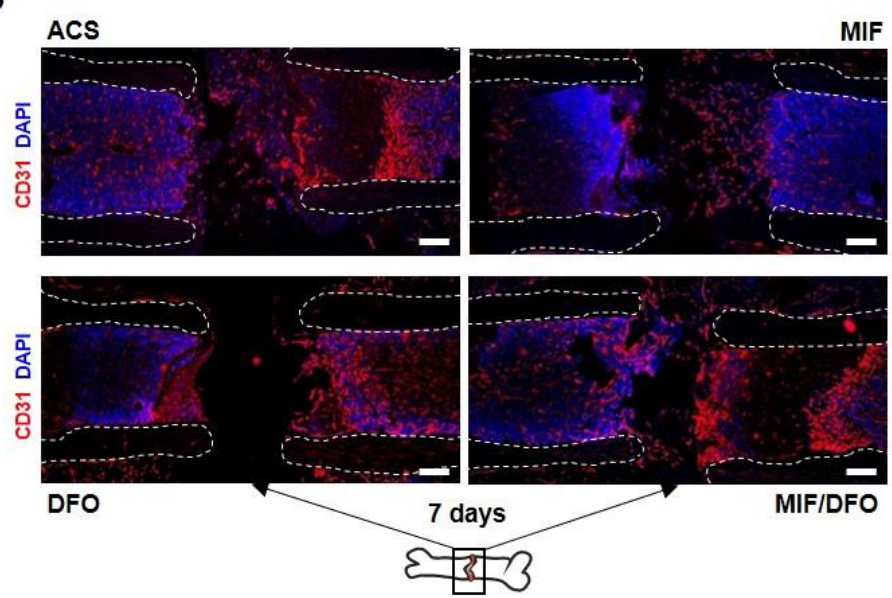

Figure S13: Revascularization in a delayed healing model under MIF, DFO and MIF/DFO treatment additional data. (A) Quantification $C D 31^{+}$stained areas and cell numbers per area normalized to the median of the ACS group (indicated as dotted line $=1$ ) and $(B)$ corresponding representative images for day 3 and $7(N=3-4)$. White dotted lines indicate cortices. Schematic bone indicates alignment of images. Scale bars $=200 \mu \mathrm{m}$. Data are shown as box plots with the median as horizontal line, interquartile range as boxes, minimum/maximum as whiskers and individual data points. Wilcoxon signed rank test was applied 
A

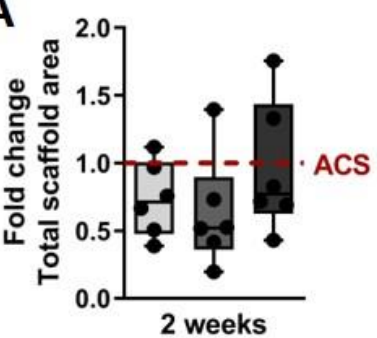

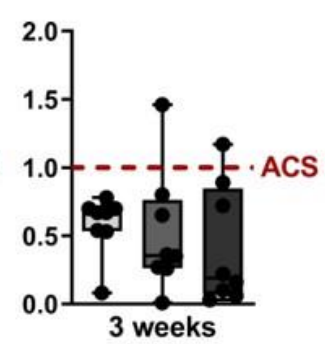

B

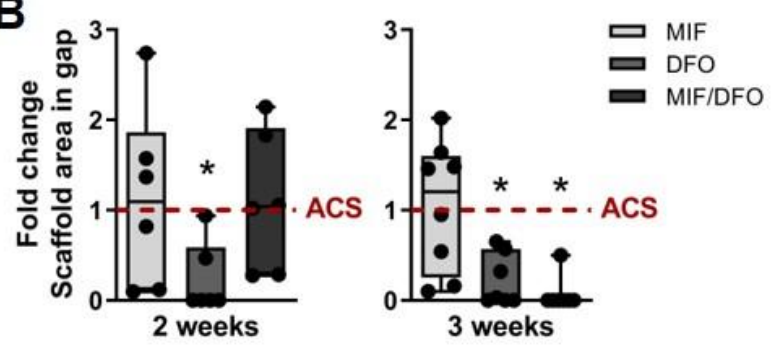

992

993

994

995

996

997

998

999

1000

Table S3: List of hMSCs that were used in the study.

\begin{tabular}{|c|c|c|c|c|c|}
\hline Donor & Age & Gender & Cultivation condition & Characterization & Experiments \\
\hline 1 & 52 & female & \multirow{5}{*}{$\begin{array}{l}\text { Expansion in normal medium: } \\
\text { DMEM plus GlutaMAX, 10\% FCS, } \\
1 \% \text { Penicillin-Streptomycin }\end{array}$} & \multirow{22}{*}{$\begin{array}{c}\text { + plastic adherent } \\
\text { + osteogenic/adipogenic } \\
\text { differentiation } \\
+ \text { CD13, CD44, CD90, CD105 } \\
\text { - CD45, CD14, CD19 }\end{array}$} & \multirow{11}{*}{ MIF/DFO titration } \\
\hline 2 & 77 & female & & & \\
\hline 3 & 70 & female & & & \\
\hline 4 & 76 & male & & & \\
\hline 5 & 73 & female & & & \\
\hline 6 & 69 & female & \multirow{6}{*}{$\begin{array}{l}\text { Osteogenic medium: } \\
\text { DMEM plus GlutaMAX, } 10 \% \text { FCS, } \\
1 \% \text { Penicillin-Streptomycin, } \\
10 \mathrm{mM} \beta \text {-glycerophosphate, } \\
\text { 10-8 M dexamethasone, } 0.1 \mathrm{mM} \mathrm{L-} \\
\text { ascorbic acid-2-phosphate }\end{array}$} & & \\
\hline 7 & 48 & male & & & \\
\hline 8 & 75 & female & & & \\
\hline 9 & 82 & male & & & \\
\hline 10 & 56 & female & & & \\
\hline 11 & 66 & male & & & \\
\hline 12 & 69 & female & \multirow{11}{*}{$\begin{array}{c}\text { Expansion in normal medium: } \\
\text { DMEM plus GlutaMAX, 20\% } \\
\text { StemMACS, 10\% (v/v) FCS, 1\% } \\
\text { Penicillin-Streptomycin } \\
\text { Osteogenic medium: } \\
\text { StemMACS OsteoDiff Medium, \% } \\
\text { Penicillin-Streptomycin }\end{array}$} & & \multirow{11}{*}{ DFO release experiments } \\
\hline 13 & 57 & male & & & \\
\hline 14 & 84 & female & & & \\
\hline 15 & 65 & male & & & \\
\hline 16 & 71 & female & & & \\
\hline 17 & 75 & female & & & \\
\hline 19 & 78 & male & & & \\
\hline 20 & 61 & female & & & \\
\hline 21 & 63 & male & & & \\
\hline 22 & 77 & male & & & \\
\hline 23 & 63 & male & & & \\
\hline
\end{tabular}
(A) Quantified total scaffold areas and (B) in the gap after 2 and 3 weeks normalized to the median of the ACS group (indicated as dotted line $=1) .(N=6-8)$. Data are shown as box plots with the median as horizontal line, interquartile range as boxes, minimum/maximum as whiskers and individual data points. Wilcoxon signed rank test was applied to determine difference against the ACS control group (hypothetical value $=1$ ) and Kruskal Wallis test with Dunn's multiple comparison test was used to compare groups. *P $<0.05$. 